\title{
Calculating three-loop diagrams in heavy quark effective theory with integration-by-parts recurrence relations
}

\author{
Andrey G. Grozin \\ Institut für Theoretische Teilchenphysik \\ Universität Karlsruhe \\ E-mail: grozin@particle.uni-karlsruhe.de
}

\begin{abstract}
An algorithm for calculation of three-loop propagator diagrams in HQET, based on integration-by-parts recurrence relations, is constructed and implemented as a REDUCE package Grinder, and in Axiom.
\end{abstract}

KEYWORDS: Renormalization Regularization and Renormalons, Heavy Quarks Physics, QCD, NLO Computations. 


\section{Contents}

1. Introduction 1

2. Diagrams with lower-loop propagator insertions 2

2.1 Two-loop massless propagator diagrams 2

2.2 Two-loop HQET propagator diagrams 5

2.3 Three-loop HQET diagrams with lower-loop insertions 8

3. Proper three-loop HQET propagator diagrams 10

3.1 Diagram with two heavy-quark lines 11

3.2 Mercedez with three heavy-quark lines 15

3.3 Ladder with three heavy-quark lines 18

3.4 Non-planar diagram with three heavy-quark lines 20

3.5 Diagrams with four heavy-quark lines 24

3.6 Diagrams with five heavy-quark lines 26

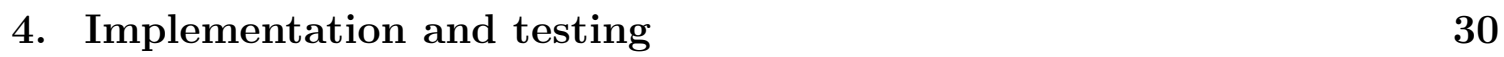

\section{Introduction}

Perturbative quantum field theory is progressing fast. New high-precision experiments require calculation of higher radiative corrections - multiloop Feynman diagrams. Recently, some calculations have been done which would seem impossible only a few years ago. This is due to the high degree of automation of the process of generation, analyses and calculation of Feynman diagrams, which is achieved via extensive use of computer algebra (see [1] for review and references). All such calculations are performed in the framework of dimensional regularization [2], i.e. diagrams are calculated as analytical functions of the space-time dimension $d=4-2 \epsilon$.

The integration-by-parts method [3] was invented for calculation of three-loop massless propagator diagrams. It is the most systematic method of those currently used, and the most appropriate for computer-algebra implementation. It was first implemented as a SCHOONSCHIP [4] package MINCER [5], and later re-implemented [6] in FORM [7] (in fact, FORM was created mainly to run MINCER). Since then, MINCER has been the engine behind most of spectacular successes of perturbative field theory. Some of these calculations, with gigabyte-size intermediate expressions, are among the largest computer-algebra calculations ever undertaken.

Integration-by-parts was used for other classes of problems, too. Many interesting physical results have been obtained with packages for calculating two-loop 
on-shell massive diagrams and three-loop vacuum diagrams with a single mass (see, e.g., [8, 9, 10]). Reduction of two-loop propagator integrals with generic masses and momentum to a finite set of bases integrals has been achieved [11]. First three-loop on-shell calculations have been done recently [12, 13].

Several years ago, an interesting new approach to heavy-quark problems in Quantum Chromodynamics has been formulated - Heavy Quark Effective Theory (HQET), see, e.g., [14, 15] for review and references. In collaboration with David Broadhurst, I applied the integration-by-parts method for calculating two-loop propagator diagrams in HQET [16]. Since then, the algorithm suggested was used in a large number of physics applications. A short review of the integration-by-parts method as applied in heavy quark physics is presented in [17.

In the present work, I apply this method for calculating three-loop propagator diagrams in HQET. Three-loop anomalous dimensions and spectral densities in HQET are necessary for a number of physics applications, such as improved extraction of the $B$ meson decay constant $f_{B}$ from lattice simulations and from QCD sum rules. HQET lagrangian does not involve mass in the leading order, and, therefore, the problem is quite similar to the massless one. My aim is to produce a reliable package for three-loop HQET calculations, which could play the same role as MINCER in massless theories. I call it Grinder. Some complication comes from the fact that there are two kinds of lines now - massless propagators and infinitely-heavy ones, and hence the number of diagram topologies is substantially larger.

In section 2, we consider three-loop HQET propagator diagrams with one- or two-loop massless or HQET propagator subdiagrams. To this end, we first recall well-known results for massless [3] and HQET [16] one- and two-loop diagrams. After that, diagrams with two-loop insertions, or with two one-loop insertions, are easily calculated. Two-loop HQET diagrams with a single one-loop insertion are dealt with in a manner similar to the plain two-loop diagrams. In section 3, we consider proper three-loop HQET propagator diagrams. Some details of implementation and testing are presented in section 4 .

\section{Diagrams with lower-loop propagator insertions}

\subsection{Two-loop massless propagator diagrams}

The one-loop massless propagator integral (figure 1) can be easily calculated by using the Feynman parameterization or Fourier transform to the coordinate space and back:

$$
\begin{aligned}
\int \frac{d^{d} k}{D_{1}^{n_{1}} D_{2}^{n_{2}}} & =i \pi^{d / 2}\left(-p^{2}\right)^{d / 2-n_{1}-n_{2}} G\left(n_{1}, n_{2}\right), \\
D_{1} & =-k^{2}, \quad D_{2}=-(k+p)^{2} \\
G\left(n_{1}, n_{2}\right) & =\frac{\Gamma\left(n_{1}+n_{2}-d / 2\right) \Gamma\left(d / 2-n_{1}\right) \Gamma\left(d / 2-n_{2}\right)}{\Gamma\left(n_{1}\right) \Gamma\left(n_{2}\right) \Gamma\left(d-n_{1}-n_{2}\right)} .
\end{aligned}
$$

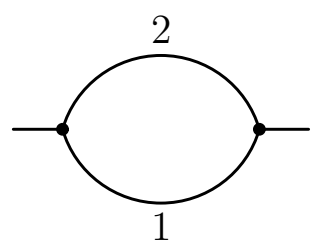

Figure 1: Oneloop massless propagator diagram. 

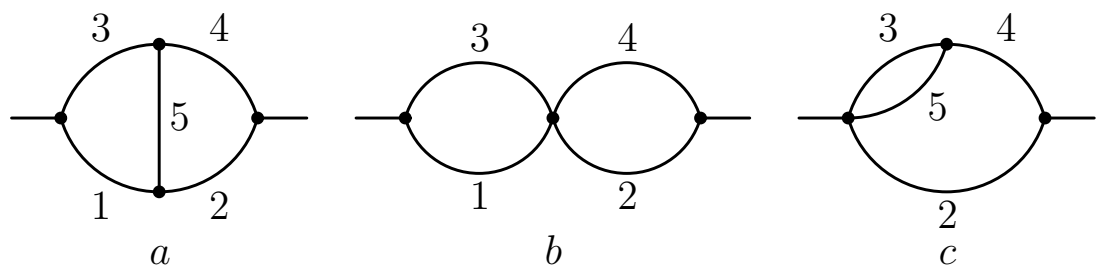

Figure 2: Two-loop massless propagator diagram

The integral with a numerator can be written as a finite sum [3]

$$
\begin{aligned}
\int \frac{P_{n}(k) d^{d} k}{D_{1}^{n_{1}} D_{2}^{n_{2}}}= & i \pi^{d / 2}\left(-p^{2}\right)^{d / 2-n_{1}-n_{2}} \times \\
& \times\left.\sum_{m} G\left(n_{1}, n_{2} ; n, m\right) \frac{\left(-p^{2}\right)^{m}}{m !}\left(-\frac{1}{4} \frac{\partial}{\partial k_{\mu}} \frac{\partial}{\partial k^{\mu}}\right)^{m} P_{n}(k)\right|_{k \rightarrow p}, \quad(2.2) \\
G\left(n_{1}, n_{2} ; n, m\right)= & \frac{\Gamma\left(n_{1}+n_{2}-m-d / 2\right) \Gamma\left(d / 2-n_{1}+n-m\right) \Gamma\left(d / 2-n_{2}+m\right)}{\Gamma\left(n_{1}\right) \Gamma\left(n_{2}\right) \Gamma\left(d-n_{1}-n_{2}+n\right)},
\end{aligned}
$$

where $P_{n}(k)$ is an arbitrary homogeneous polynomial: $P_{n}(\lambda k)=\lambda^{n} P_{n}(k)$.

We write the two-loop propagator integral (figure $2 a$ ) as

$$
\begin{aligned}
& \int \frac{d^{d} k_{1} d^{d} k_{2}}{D_{1}^{n_{1}} D_{2}^{n_{2}} D_{3}^{n_{3}} D_{4}^{n_{4}} D_{5}^{n_{5}}}=-\pi^{d}\left(-p^{2}\right)^{d-\sum n_{i}} G\left(n_{1}, n_{2}, n_{3}, n_{4}, n_{5}\right), \\
& D_{1}=-k_{1}^{2}, \quad D_{2}=-k_{2}^{2}, \quad D_{3}=-\left(k_{1}+p\right)^{2}, \quad D_{4}=-\left(k_{2}+p\right)^{2}, \\
& D_{5}=-\left(k_{1}-k_{2}\right)^{2} .
\end{aligned}
$$

It is symmetric with respect to $1 \leftrightarrow 2,3 \leftrightarrow 4$, and also $1 \leftrightarrow 3,2 \leftrightarrow 4$. If one of the indices is zero, it can be easily calculated using (2.1) (figure [b $2 b$ )

$$
\begin{aligned}
& G\left(n_{1}, n_{2}, n_{3}, n_{4}, 0\right)=G\left(n_{1}, n_{3}\right) G\left(n_{2}, n_{4}\right), \\
& G\left(0, n_{2}, n_{3}, n_{4}, n_{5}\right)=G\left(n_{3}, n_{5}\right) G\left(n_{2}, n_{4}+n_{3}+n_{5}-d / 2\right)
\end{aligned}
$$

(and symmetric relations).

Applying the operators $\partial_{1} \cdot\left(k_{1}-k_{2}\right)$ and $\partial_{1} \cdot k_{1}$ (where $\left.\partial_{i}=\partial / \partial k_{i}\right)$ to the integrand of (2.3), we obtain the recurrence relations for $G\left(n_{1}, n_{2}, n_{3}, n_{4}, n_{5}\right)$ (known as triangle relations [3])

$$
\begin{array}{r}
{\left[d-n_{1}-n_{3}-2 n_{5}+n_{1} \mathbf{1}^{+}\left(\mathbf{2}^{-}-\mathbf{5}^{-}\right)+n_{3} \mathbf{3}^{+}\left(\mathbf{4}^{-}-\mathbf{5}^{-}\right)\right] G=0} \\
{\left[d-n_{3}-n_{5}-2 n_{1}+n_{3} \mathbf{3}^{+}\left(1-\mathbf{1}^{-}\right)+n_{5} \mathbf{5}^{+}\left(\mathbf{2}^{-}-\mathbf{1}^{-}\right)\right] G=0}
\end{array}
$$

where, for example,

$$
\mathbf{1}^{ \pm} G\left(n_{1}, n_{2}, n_{3}, n_{4}, n_{5}\right)=G\left(n_{1} \pm 1, n_{2}, n_{3}, n_{4}, n_{5}\right) .
$$




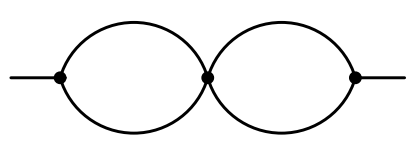

$a$

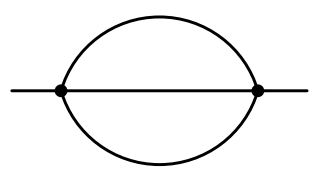

$b$

Figure 3: Basis two-loop massless propagator integrals

Of course, more relations are obtained by symmetry. Another interesting relation is derived by applying the operator $\frac{\partial}{\partial p} \cdot\left(k_{2}+p\right)$. Substituting the general form of the relevant vector integral, we arrive at

$$
\begin{array}{r}
{\left[\frac{1}{2} d+n_{4}-n_{1}-n_{2}-n_{5}+\left(\frac{3}{2} d-n_{1}-n_{2}-n_{3}-n_{4}-n_{5}\right)\left(\mathbf{4}^{-}-\mathbf{2}^{-}\right)+\quad\right.} \\
\left.+n_{3} \mathbf{3}^{+}\left(\mathbf{4}^{-}-\mathbf{5}^{-}\right)\right] G=0 .
\end{array}
$$

This formula was derived long ago by S.A. Larin in his M.Sc. thesis [18] (again, similar relations follow by symmetries).

If indices of two adjacent lines are non-positive integers, the integral contains a no-scale vacuum subdiagram and hence vanishes. The cases with zero indices are given by (2.4), (2.5). When $n_{5}<0$ and $n_{3} \neq 1, n_{5}$ can be raised by (2.9); the cases $n_{1} \neq 1, n_{2} \neq 1, n_{4} \neq 1$ are symmetric. The case $n_{5}<0, n_{1}=n_{1}=n_{3}=n_{4}=1$ is handled by

$$
\begin{aligned}
{\left[\left(d-2 n_{5}-4\right) \mathbf{5}^{+}+2\left(d-n_{5}-3\right)\right] G\left(1,1,1,1, n_{5}\right)=} \\
=2 \mathbf{1}^{+}\left(\mathbf{3}^{-}-\mathbf{2}^{-} \mathbf{5}^{+}\right) G\left(1,1,1,1, n_{5}\right),
\end{aligned}
$$

which follows from (2.6) and (2.7) at $n_{1}=n_{2}=n_{3}=n_{4}=1$ (note that the terms in the right-hand side of (2.10) are trivial for any $n_{5}$; for $n_{5}<0$, they vanish). When $n_{2}<0$, it can be raised by (2.9); the cases $n_{1}<0, n_{3}<0, n_{4}<0$ are symmetric.

We are left with the most important situation when all the indices are positive. Applying (2.6), we reduce $n_{2}, n_{4}, n_{5}$ until one of them vanishes. Then (2.4) and (2.5) apply. If $\max \left(n_{1}, n_{3}\right)<\max \left(n_{2}, n_{4}\right)$, it is more efficient to lower $n_{1}, n_{3}, n_{5}$.

All one-loop integrals (figure (1) with integer $n_{1,2}$ are proportional to $G_{1}=$ $G(1,1)$, the coefficient being a rational function of $d$. All two-loop integrals with integer indices reduce to $G_{1}^{2}$ (figure $3 a$ ) and $G_{2}=G(0,1,1,0,1$ ) (figure $3 b$ ), with rational coefficients. Here

$$
G_{n}=\frac{1}{\left(n+1-n \frac{d}{2}\right)_{n}\left((n+1) \frac{d}{2}-2 n-1\right)_{n}} \frac{\Gamma(1+n \epsilon) \Gamma^{n+1}(1-\epsilon)}{\Gamma(1-(n+1) \epsilon)},
$$

where $(x)_{n}=\Gamma(x+n) / \Gamma(x)$ is the Pochhammer symbol. 


\subsection{Two-loop HQET propagator diagrams}

The one-loop HQET propagator integral (figure 廿) can be easily calculated by using the modified Feynman parameterization (see, e.g., [16, 17]), or Fourier transform to the coordinate space and back:

$$
\begin{aligned}
\int \frac{d^{d} k}{D_{1}^{n_{1}} D_{2}^{n_{2}}} & =i \pi^{d / 2}(-2 \omega)^{d-2 n_{2}} I\left(n_{1}, n_{2}\right), \\
D_{1} & =\frac{(k+p) \cdot v}{\omega}, \quad D_{2}=-k^{2}, \\
I\left(n_{1}, n_{2}\right) & =\frac{\Gamma\left(n_{1}+2 n_{2}-d\right) \Gamma\left(d / 2-n_{2}\right)}{\Gamma\left(n_{1}\right) \Gamma\left(n_{2}\right)}
\end{aligned}
$$

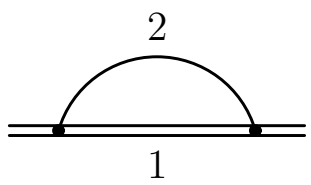

Figure 4: Oneloop HQET propagator diagram.

(here $v$ is 4 -velocity of the heavy quark, $v^{2}=1$, and $\omega=p \cdot v$ is the residual energy). Similarly to (2.2), we obtain

$$
\begin{aligned}
\int \frac{P_{n}(k) d^{d} k}{D_{1}^{n_{1}} D_{2}^{n_{2}}}= & i \pi^{d / 2}(-2 \omega)^{d-2 n_{2}} \times \\
& \times\left.\sum_{m} I\left(n_{1}, n_{2} ; n, m\right) \frac{(-2 \omega)^{2 m}}{m !}\left(-\frac{1}{4} \frac{\partial}{\partial k_{\mu}} \frac{\partial}{\partial k^{\mu}}\right)^{m} P_{n}(k)\right|_{k \rightarrow 2 \omega v}, \\
I\left(n_{1}, n_{2} ; n, m\right)= & \frac{\Gamma\left(n_{1}+2 n_{2}-n-d\right) \Gamma\left(d / 2-n_{2}+n-m\right)}{\Gamma\left(n_{1}\right) \Gamma\left(n_{2}\right)} .
\end{aligned}
$$

There are two topologies of two-loop propagator HQET diagrams (figure $5 a, b$ ). We write the first of them as

$$
\begin{aligned}
\int \frac{d^{d} k_{1} d^{d} k_{2}}{D_{1}^{n_{1}} D_{2}^{n_{2}} D_{3}^{n_{3}} D_{4}^{n_{4}} D_{5}^{n_{5}}} & =-\pi^{d}(-2 \omega)^{2\left(d-n_{3}-n_{4}-n_{5}\right)} I\left(n_{1}, n_{2}, n_{3}, n_{4}, n_{5}\right), \\
D_{1} & =\frac{\left(k_{1}+p\right) \cdot v}{\omega}, \quad D_{2}=\frac{\left(k_{2}+p\right) \cdot v}{\omega}, \\
D_{3} & =-k_{1}^{2}, \quad D_{4}=-k_{2}^{2}, \quad D_{5}=-\left(k_{1}-k_{2}\right)^{2} .
\end{aligned}
$$

It is symmetric with respect to $1 \leftrightarrow 3,2 \leftrightarrow 4$. If one of the indices is zero, it can be easily calculated using (2.12) and (2.1) (figure 5c,d,e)

$$
\begin{aligned}
& I\left(n_{1}, n_{2}, n_{3}, n_{4}, 0\right)=I\left(n_{1}, n_{3}\right) I\left(n_{2}, n_{4}\right), \\
& I\left(0, n_{2}, n_{3}, n_{4}, n_{5}\right)=G\left(n_{3}, n_{5}\right) I\left(n_{2}, n_{4}+n_{3}+n_{5}-d / 2\right), \\
& I\left(n_{1}, n_{2}, 0, n_{4}, n_{5}\right)=I\left(n_{1}, n_{5}\right) I\left(n_{2}+n_{1}+2 n_{5}-d, n_{4}\right)
\end{aligned}
$$

(and symmetric relations).

Applying the operators $\partial_{1} \cdot\left(k_{1}-k_{2}\right), \partial_{1} \cdot k_{1}$ and $\partial_{1} \cdot v$ to the integrand of (2.14), we obtain 16]

$$
\begin{aligned}
{\left[d-n_{1}-n_{3}-2 n_{5}+n_{1} \mathbf{1}^{+} \mathbf{2}^{-}+n_{3} \mathbf{3}^{+}\left(\mathbf{4}^{-}-\mathbf{5}^{-}\right)\right] I } & =0 \\
{\left[d-n_{1}-n_{5}-2 n_{3}+n_{1} \mathbf{1}^{+}+n_{5} \mathbf{5}^{+}\left(\mathbf{4}^{-}-\mathbf{3}^{-}\right)\right] I } & =0 \\
{\left[-2 n_{1} \mathbf{1}^{+}+n_{3} \mathbf{3}^{+}\left(\mathbf{1}^{-}-1\right)+n_{5} \mathbf{5}^{+}\left(\mathbf{1}^{-}-\mathbf{2}^{-}\right)\right] I } & =0 .
\end{aligned}
$$



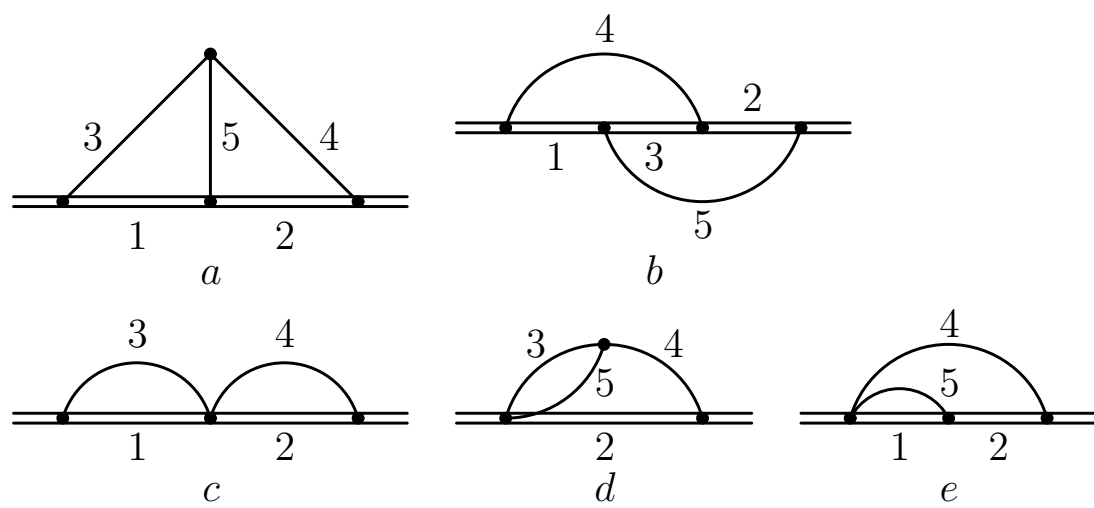

Figure 5: Two-loop HQET propagator diagram

Of course, more relations are obtained by symmetry. Applying $\omega \frac{d}{d \omega}$ and using homogeneity in $\omega$, we obtain

$$
\left[2\left(d-n_{3}-n_{4}-n_{5}\right)-n_{1}-n_{2}+n_{1} \mathbf{1}^{+}+n_{2} \mathbf{2}^{+}\right] I=0
$$

which is nothing but the sum of (2.19) and its mirror-symmetric. Subtracting the $2^{-}$shifted version of (2.21) from (2.18), we obtain the most useful relation [16]

$$
\begin{gathered}
{\left[d-n_{1}-n_{2}-n_{3}-2 n_{5}+1-\right.} \\
\left.-\left(2\left(d-n_{3}-n_{4}-n_{5}\right)-n_{1}-n_{2}+1\right) 2^{-}+n_{3} 3^{+}\left(\mathbf{4}^{-}-\mathbf{5}^{-}\right)\right] I=0,
\end{gathered}
$$

which lowers $n_{2}, n_{4}, n_{5}$, and does not raise heavy-quark indices.

If indices of two adjacent lines are non-positive integers, the integral contains a no-scale vacuum subdiagram and hence vanishes. The cases with zero indices are given by (2.15), (2.16) and (2.17). When $n_{2}<0$, it can be raised by (2.22); the case $n_{1}<0$ is symmetric. Similarly, if $n_{3}<0$, it can be raised by $(2.22)$; the case $n_{4}<0$ is symmetric. When $n_{5}<0$ and $n_{3} \neq 1, n_{5}$ can be raised by (2.22) (the case $n_{4} \neq 1$ is symmetric); when $n_{5}<0$ and $n_{1} \neq 1, n_{5}$ can be raised by (2.19) (the case $n_{2} \neq 1$ is symmetric). The case $n_{5}<0, n_{1}=n_{1}=n_{3}=n_{4}=1$ is handled by

$$
\begin{aligned}
& {\left[\left(d-2 n_{5}-4\right) \mathbf{5}^{+}-2\left(d-n_{5}-3\right)\right] I\left(1,1,1,1, n_{5}\right)=} \\
& \quad=\left[\left(2 d-2 n_{5}-7\right) \mathbf{1}^{-} \mathbf{5}^{+}-\mathbf{3}^{-} \mathbf{4}^{+} \mathbf{5}^{-}+\mathbf{1}^{-} \mathbf{3}^{+}\right] I\left(1,1,1,1, n_{5}\right)
\end{aligned}
$$

which follows from (2.18), (2.19) and (2.20) at $n_{1}=n_{2}=n_{3}=n_{4}=1$ (note that the terms on the right-hand side of (2.23) are trivial for any $n_{5}$; for $n_{5}<0$, they vanish).

We are left with the most important situation when all the indices are positive. Applying (2.22), we reduce $n_{2}, n_{4}, n_{5}$ until one of them vanishes. Then (2.15), (2.16) and (2.17) apply. If $\max \left(n_{1}, n_{3}\right)<\max \left(n_{2}, n_{4}\right)$, it is more efficient to lower $n_{1}, n_{3}, n_{5}$.

In the second topology (figure $5 b$ ), three heavy-quark denominators depend on only two variables $k_{1,2} \cdot v$, hence they are linearly dependent. Therefore, there is one 
scalar product which cannot be expressed via the denominators. Let's define the integral

$$
\begin{array}{lll}
\int \frac{N^{n_{0}} d^{d} k_{1} d^{d} k_{2}}{D_{1}^{n_{1}} D_{2}^{n_{2}} D_{3}^{n_{3}} D_{4}^{n_{4}} D_{5}^{n_{5}}}=-\pi^{d}(-2 \omega)^{2\left(d+n_{0}-n_{4}-n_{5}\right)} J\left(n_{1}, n_{2}, n_{3}, n_{4}, n_{5} ; n_{0}\right), \\
D_{1}=\frac{\left(k_{1}+p\right) \cdot v}{\omega}, & D_{2}=\frac{\left(k_{2}+p\right) \cdot v}{\omega}, & D_{3}=\frac{\left(k_{1}+k_{2}+p\right) \cdot v}{\omega}, \\
D_{4}=-k_{1}^{2}, & D_{5}=-k_{2}^{2}, & N=2 k_{1} \cdot k_{2}
\end{array}
$$

(it is symmetric with respect to $1 \leftrightarrow 2,4 \leftrightarrow 5$ ). Noting that $D_{1}+D_{2}-D_{3}=1$, we immediately have [16]

$$
\left(1-\mathbf{1}^{-}-2^{-}+3^{-}\right) J=0 .
$$

Applying $\partial_{1} \cdot k_{1}, \partial_{1} \cdot k_{2}$ and $\partial_{1} \cdot v$ to the integrand, we have

$$
\begin{aligned}
& {\left[d+n_{0}-n_{1}-n_{3}-2 n_{4}+n_{1} \mathbf{1}^{+}+n_{3} \mathbf{3}^{+} \mathbf{2}^{-}\right] J=0} \\
& {\left[n_{1}-n_{3}-n_{1} \mathbf{1}^{+} \mathbf{3}^{-}+n_{3} \mathbf{3}^{+} \mathbf{1}^{-}+n_{4} \mathbf{4}^{+} \mathbf{0}^{+}-2 n_{0} \mathbf{0}^{-} \mathbf{5}^{-}\right] J=0} \\
& {\left[-2 n_{1} \mathbf{1}^{+}-2 n_{3} \mathbf{3}^{+}+n_{4} \mathbf{4}^{+}\left(\mathbf{1}^{-}-1\right)+n_{0} \mathbf{0}^{-}\left(\mathbf{2}^{-}-1\right)\right] J=0 .}
\end{aligned}
$$

Homogeneity in $\omega$ gives $\left[2\left(d+n_{0}-n_{4}-n_{5}\right)-n_{1}-n_{2}-n_{3}+n_{1} \mathbf{1}^{+}+n_{2} \mathbf{2}^{+}+n_{3} \mathbf{3}^{+}\right] J=0$, which is nothing but the sum of (2.26) and its mirror-symmetric. The boundary values of the integral (figure $5 c, e$ ) are

$$
\begin{aligned}
& J\left(n_{1}, n_{2}, 0, n_{4}, n_{5} ; n_{0}\right)=\left(5^{-}-\mathbf{3}^{-}-4^{-}\right)^{n_{0}} I\left(n_{1}, n_{2}, n_{4}, n_{5}, 0\right) \\
& J\left(0, n_{2}, n_{3}, n_{4}, n_{5} ; n_{0}\right)=\left(4^{-}-3^{-}+5^{-}\right)^{n_{0}} I\left(n_{3}, n_{2}, 0, n_{5}, n_{4}\right)
\end{aligned}
$$

and the symmetric relation for $n_{2}=0$. If $n_{0}=0$,

$$
\begin{aligned}
& J\left(n_{1}, n_{2}, 0, n_{4}, n_{5}\right)=I\left(n_{1}, n_{4}\right) I\left(n_{2}, n_{5}\right) \\
& J\left(0, n_{2}, n_{3}, n_{4}, n_{5}\right)=I\left(n_{3}, n_{4}\right) I\left(n_{2}+n_{3}+2 n_{4}-d, n_{5}\right) .
\end{aligned}
$$

If $n_{4} \leq 0$, or $n_{5} \leq 0$, or two adjacent heavy-quark indices are non-positive, the integral vanishes. If any of $n_{1}, n_{2}, n_{3}$ is negative, it can be raised by (2.25). If all of them are positive, we use (2.25) to lower $n_{1}, n_{2}$ or $n_{3}$, until one of these indices vanish.

Instead of using (2.29), (2.30) when $n_{0}>0$, we could proceed in another way. If $n_{4}>1$, we lower both $n_{0}$ and $n_{4}$ using (2.27) (the case $n_{5}>1$ is symmetric). We are left with $J\left(n_{1}, n_{2}, 0,1,1 ; n_{0}\right)$ and $J\left(0, n_{2}, n_{3}, 1,1 ; n_{0}\right)$. In the first case, if $n_{1}>1$, we lower it using (2.28) (the case $n_{2}>1$ is symmetric). In the second case, if $n_{3}>1$, we lower it using (2.28); if $n_{2}>1$, we lower it using the difference of (2.28) and its mirror-symmetric. The two remaining cases (figure 5 $5 c, e$ ) are easily evaluated using (2.13):

$$
\begin{aligned}
& J\left(1,1,0,1,1 ; n_{0}\right)=(-1)^{n_{0}} I\left(1,1 ; n_{0}, 0\right) I(1,1) \\
& J\left(0,1,1,1,1 ; n_{0}\right)=I\left(1,1 ; n_{0}, 0\right) \sum_{l=0}^{n_{0}} \frac{(-1)^{l} n_{0} !}{l !\left(n_{0}-l\right) !} I\left(4-2 n_{0}+l-d, 1\right)
\end{aligned}
$$




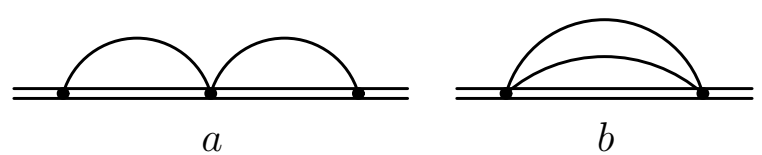

Figure 6: Basis two-loop HQET propagator integrals
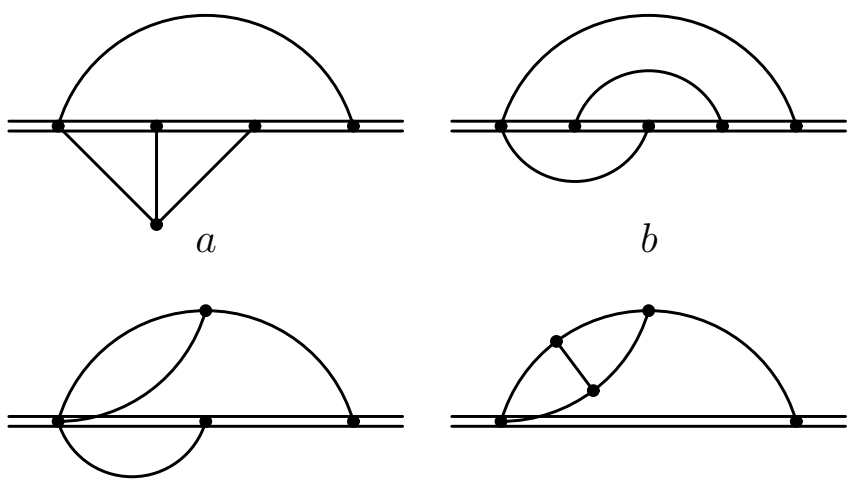

$c$

$d$

Figure 7: Three-loop HQET propagator diagrams with a two-loop propagator insertion, or with two one-loop insertions

All one-loop integrals (figure 4) with integer $n_{1,2}$ are proportional to $I_{1}=I(1,1)$, the coefficient being a rational function of $d$. All two-loop integrals with integer indices reduce to $I_{1}^{2}$ (figure $6 a$ ) and $I_{2}=I(0,1,1,0,1$ ) (figure 6 $b$ ), with rational coefficients. Here

$$
I_{n}=\frac{1}{(1-n(d-2))_{2 n}} \Gamma(1+2 n \epsilon) \Gamma^{n}(1-\epsilon) .
$$

\subsection{Three-loop HQET diagrams with lower-loop insertions}

Diagrams with a two-loop propagator insertion, or with two one-loop insertions, figure 7, are trivially calculated by multiplying the relevant insertion[s] by the one-loop HQET integral with non-integer indices, whose values are obvious by dimensionality.

Next we consider the diagrams obtained from the two-loop HQET diagram of figure $5 a$ by adding a single one-loop propagator insertion (figure 8). They are equal to the product of the corresponding one-loop integral and the integral of figure $5 a$ with one non-integer index. All relations derived for the diagram of figure $5 a$ are valid; however, the non-integer index changes the strategy of their application.

Let's consider the case of figure $8 a$ with a non-integer $n_{3}$. When $n_{5}<0$, it can always be raised by (2.22). When $n_{2}<0$, it can be raised by (2.22), too; when $n_{1}<0$, it can be raised by the mirror-symmetric relation. When $n_{4}<0$, it can always be raised by (2.22), too. Finally, when all indices $n_{1}, n_{2}, n_{4}, n_{5}$ are positive, we use (2.22) to lower $n_{2}, n_{4}$ or $n_{5}$, until (2.15), (2.16) and (2.17) are reached.

In the case of figure $8 \mathrm{~b}, n_{1}$ is non-integer. When $n_{5}<0$ and $n_{3} \neq 1$, we can raise $n_{5}$ by $(2.22)$ (the case $n_{4} \neq 1$ is symmetric); when $n_{5}<0$ and $n_{2} \neq 1$, we can lower 


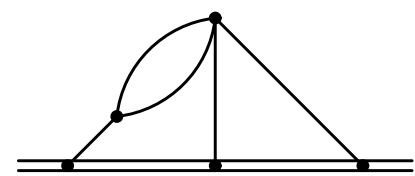

$a$

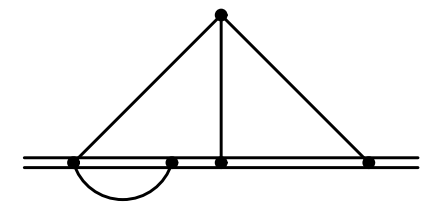

$b$

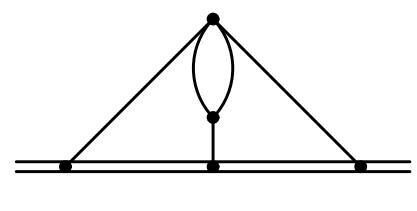

$c$

Figure 8: The diagram of figure $5 a$ with a single one-loop insertion

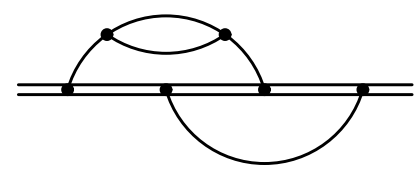

$a$

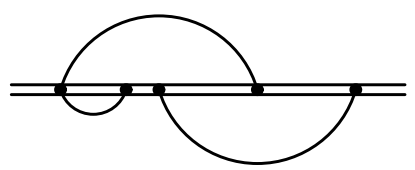

$b$

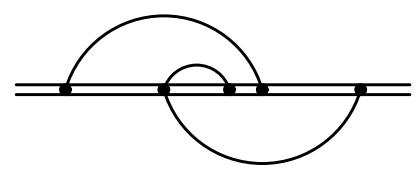

$c$

Figure 9: The diagram of figure $5 b$ with a single one-loop insertion

$n_{2}$ by the relation symmetric to (2.19); when $n_{5}<0$ and $n_{2}=n_{3}=n_{4}=1$, we can use the relation

$$
\left[2\left(d-n_{5}-3\right)-\mathbf{4}^{+}-n_{5} \mathbf{5}^{+} \mathbf{1}^{-}\right] I\left(n_{1}, 1,1,1, n_{5}\right)=0
$$

which follows from (2.20) and (2.19), and then the term with $4^{+}$can be treated as above. When $n_{2}<0$, it can be raised by (2.22). When $n_{3}<0$ and $n_{4} \neq 1$, we can raise $n_{3}$ by the relation symmetric to (2.22); when $n_{3}<0$ and $n_{5} \neq 1$, we can raise $n_{3}$ by (2.19); when $n_{3}<0$ and $n_{2} \neq 1$, we lower $n_{2}$ by (2.21); finally, when $n_{3}<0$ and $n_{2}=n_{4}=n_{5}=1$, we use (2.22) to get a trivial term with $n_{2}=0$. When $n_{4}<0$ and $n_{3} \neq 1$, we can raise $n_{4}$ by (2.22); when $n_{4}<0$ and $n_{5} \neq 1$, we can raise $n_{4}$ by (2.19); when $n_{4}<0$ and $n_{2} \neq 1$, we lower $n_{2}$ by (2.21); finally, when $n_{4}<0$ and $n_{2}=n_{3}=n_{5}=1$, we use (2.20) to raise $n_{3}$ or $n_{5}$, and proceed as above. Finally, when all indices $n_{2}, n_{3}, n_{4}, n_{5}$ are positive, we use (2.22) to lower $n_{2}, n_{4}$ or $n_{5}$, until (2.15), 2.16) and (2.17) are reached.

In the case of figure $8 c, n_{5}$ is non-integer. When $n_{2}<0$, it can be raised by (2.22); the case $n_{1}<0$ is symmetric. When $n_{3}<0$, it can be raised by (2.19); the case $n_{4}<0$ is symmetric. When $n_{1}>1$, it can be lowered by (2.19); the case $n_{2}>1$ is symmetric. When $n_{3}>1$, it can be lowered by (2.20), with a trivial additional term having $n_{1}=0$; the case $n_{4}>1$ is symmetric. We are left with $n_{1}=n_{2}=n_{3}=n_{4}=1$; the relation (2.23) can be used to lower or raise $n_{5}$, with trivial additional terms. An integral with some specific value of $n_{5}$ (of the form integer plus $\epsilon$ ) has to be considered as a new basis element. This integral has been calculated, exactly in $d$ dimensions, in [19] in terms of ${ }_{3} F_{2}$ hypergeometric functions, using the Hegenbauer polynomial technique in the coordinate space [20].

Finally, we consider the diagrams obtained from the two-loop HQET diagram of figure $5 b$ by adding a single one-loop propagator insertion (figure 9). The diagram of figure $9 a$, with a non-integer $n_{4}$ is calculated exactly as the two-loop one. 
In the case of figure $9 b, n_{1}$ is non-integer. When $n_{3}<0$ or $n_{2}<0$, they can be raised by (2.25). In the case of positive indices, we use

$$
\left[d-n_{3}-2 n_{4}+n_{1} \mathbf{1}^{+}\left(\mathbf{2}^{-}-\mathbf{3}^{-}\right)+n_{3} \mathbf{3}^{+} \mathbf{2}^{-}\right] J=0
$$

(which follows from (2.26) and (2.25)). It either lowers $n_{2}+n_{3}$, or, at a fixed $n_{2}+n_{3}$, lowers $n_{2}$. Therefore, sooner or later, we reach (2.29) and (2.30).

In the case of figure $9 \mathrm{c}, n_{3}$ is non-integer. If $n_{1}<0$ or $n_{2}<0$, they can be raised by (2.25). When $n_{4}>1$, we can lower it or $n_{1}$ by (2.28); the case $n_{5}>1$ is symmetric. When $n_{1}>1$, it can be lowered by (2.26); the case $n_{2}>1$ is symmetric. We are left with $n_{1}=n_{2}=n_{4}=n_{5}=1$; the relation (2.25) can be used to lower or raise $n_{3}$, with trivial additional terms. An integral with some specific value of $n_{3}$ (of the form integer plus $2 \epsilon$ ) has to be considered as a new basis element.

It is not difficult to calculate $J\left(1,1, n_{3}, n_{4}, n_{5}\right)$ for arbitrary $n_{3,4,5}$ (not necessarily integer) in the coordinate space:

$$
\begin{aligned}
J\left(1,1, n, n_{1}, n_{2}\right) & =\frac{\Gamma\left(n-2\left(d-n_{1}-n_{2}-1\right)\right) \Gamma\left(d / 2-n_{1}\right) \Gamma\left(d / 2-n_{2}\right)}{\Gamma(n) \Gamma\left(n_{1}\right) \Gamma\left(n_{2}\right)} J \\
J & =t^{2\left(d-n_{1}-n_{2}\right)-n-1} \int_{0<t_{1}<t_{2}<t} d t_{1} d t_{2} t_{2}^{2 n_{1}-d}\left(t-t_{1}\right)^{2 n_{2}-d}\left(t_{2}-t_{1}\right)^{n-1} \\
& =\frac{1}{n\left(2 n_{1}+n+1-d\right)}{ }_{3} F_{2}\left(\begin{array}{c}
1, d-2 n_{2}, 2 n_{1}+n+1-d \\
n+1,2 n_{1}+n+2-d
\end{array} \mid 1\right) \cdot(2 .
\end{aligned}
$$

All diagrams considered in this section are particular cases of the generic threeloop topologies, which will be discussed in section 3, when some lines are shrunk (i.e., some indices vanish). Therefore, we don't consider diagrams with numerators here: numerators should be dealt with in the context of generic topologies, and the formulae of this section are used only as boundary values for the corresponding recurrence relations, after elimination of numerators.

All three-loop HQET propagator integrals with lower-loop propagator insertions are linear combinations of 7 basis integrals (figure 10 $a-g$ ), coefficients being rational functions of $d$. The basis integrals of figure 10 $a\left(I_{1}^{3}\right)$, figure 10 $b\left(I_{1} I_{2}\right)$, figure 10 $c\left(I_{3}\right)$, figure $10 d\left(I_{3} I_{1}^{2} / I_{2}\right)$, and figure $10 e\left(I_{3} G_{1}^{2} / G_{2}\right)$ are known exactly in $d$ dimensions in terms of $\Gamma$ functions. Those of figure $10 \mathrm{f}, g$ contain hypergeometric ${ }_{3} F_{2}$ functions of the unit argument. Several terms of their expansion in $\epsilon$ can be obtained using the methods which were recently developed in [21]. As we shall see in section 3, there is only one additional basis integral, figure $10 h$.

\section{Proper three-loop HQET propagator diagrams}

In the massless case [3], there are only 3 topologies of proper three-loop propagator diagrams: Mercedez, Ladder, and Non-planar (plus their reduced forms obtained by shrinking some lines). Now we have 10 topologies instead (figure 11). Each of 

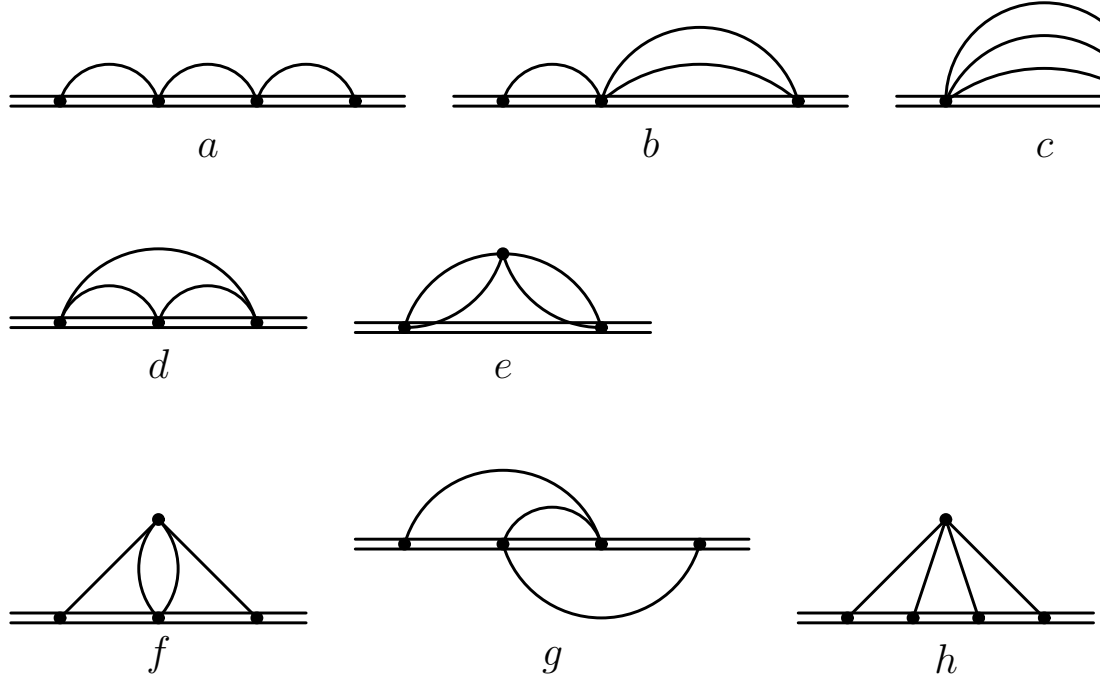

Figure 10: Basis tree-loop HQET propagator integrals

them has 8 propagators. In the diagrams with 4 heavy-quark lines (figure $11 e^{-}$ $g$ ), there is one linear dependence between their denominators; with 5 heavy-quark lines (figure $11,-j)-2$ dependences. There are 9 independent scalar products of 3 loop momenta $k_{1,2,3}$ and the 4 -velocity $v$. Therefore, in the diagrams with two or three heavy-quark lines (figure 11 $a-d$ ), there is one scalar product in the numerator which cannot be cancelled against the denominators; with 4 heavy-quark lines (figure 11 $e-g$ ) — two scalar products; with 5 heavy-quark lines (figure $11 h-j$ ) - three scalar products.

When calculating these diagrams using recurrence relations, some indices may vanish. This corresponds to shrinking the corresponding lines. In some cases, this results in diagrams with lower-loop propagator insertions, which were calculated in section 2. The diagrams of figure 12 are still non-trivial.

\subsection{Diagram with two heavy-quark lines}

Let's consider the diagram of figure 11 a first. We define

$$
\begin{aligned}
& \int \frac{N^{n_{0}} d^{d} k_{1} d^{d} k_{2} d^{d} k_{3}}{D_{1}^{n_{1}} D_{2}^{n_{2}} D_{3}^{n_{3}} D_{4}^{n_{4}} D_{5}^{n_{5}} D_{6}^{n_{6}} D_{7}^{n_{7}} D_{8}^{n_{8}}}= \\
& =-i \pi^{3 d / 2}(-2 \omega)^{3 d+2 n_{0}-2 \sum_{i=3}^{8} n_{i}} I_{a}\left(n_{1}, n_{2}, n_{3}, n_{4}, n_{5}, n_{6}, n_{7}, n_{8} ; n_{0}\right) \text {, } \\
& N=\frac{k_{3} \cdot v}{\omega}, \quad D_{1}=\frac{\left(k_{1}+p\right) \cdot v}{\omega}, \quad D_{2}=\frac{\left(k_{2}+p\right) \cdot v}{\omega} \text {, } \\
& D_{3}=-\stackrel{\omega}{k_{1}^{2}}, \quad D_{4}=-k_{2}^{2}, \stackrel{\omega}{D_{6}}, \quad D_{5}=-\left(k_{1}-k_{2}\right)^{2} \text {, } \\
& D_{6}=-k_{3}^{3}, \quad D_{7}=-\left(k_{3}+k_{1}\right)^{2}, \quad D_{8}=-\left(k_{3}+k_{2}\right)^{2} \text {. }
\end{aligned}
$$

This integral is mirror-symmetric with respect to $1 \leftrightarrow 2,3 \leftrightarrow 4,7 \leftrightarrow 8$. It vanishes when the indices of the following groups of lines are non-positive: 12, 67, 68, 78, 375, $485,315,425,364,137,248,157$, or 258. 

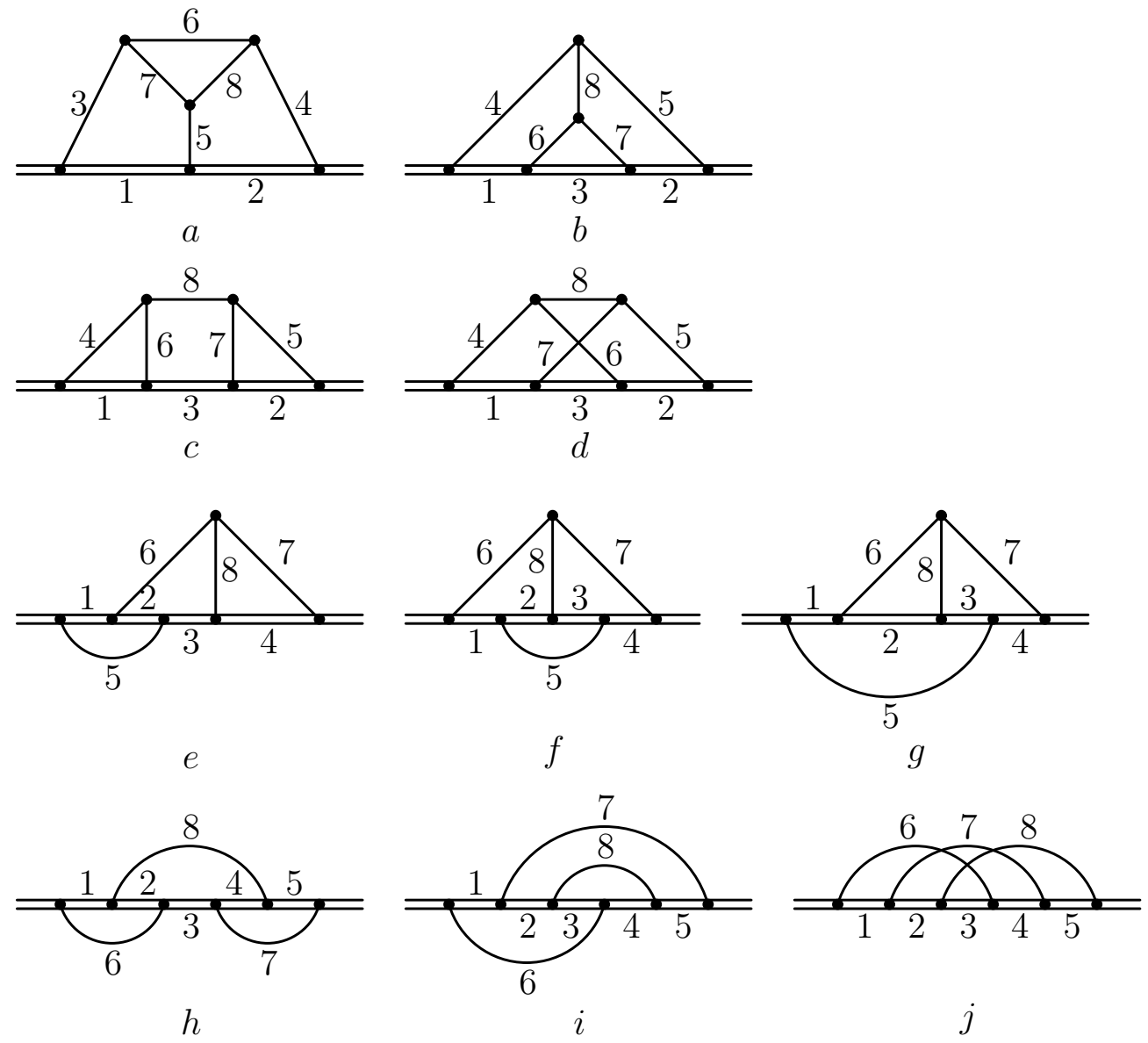

Figure 11: Topologies of proper three-loop HQET propagator diagrams: Mercedez $(a, b$, $f, g, i)$, Ladder $(c, e, h)$, Non-planar $(d, j)$
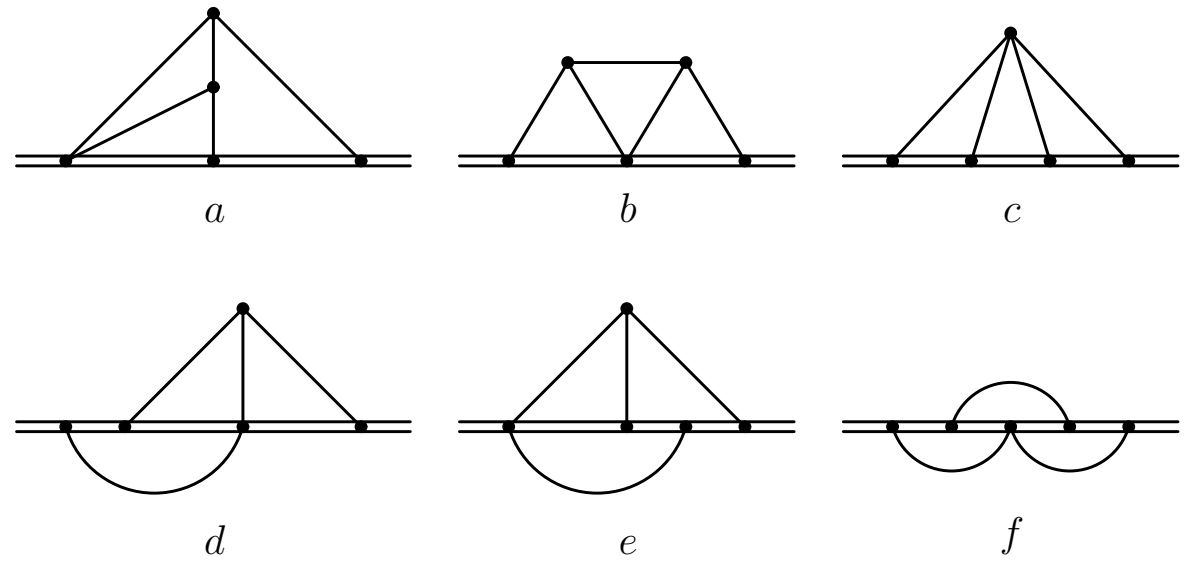

Figure 12: Topologies of non-trivial three-loop diagrams with a shrunk line 
First we are going to get rid of the numerator. When $n_{0}>0$ and $n_{7} \neq 1$, we can lower $n_{0}$ by

$$
\left[-2 n_{1} \mathbf{1}^{+}+n_{3} \mathbf{3}^{+}\left(\mathbf{1}^{-}-1\right)+n_{5} \mathbf{5}^{+}\left(\mathbf{1}^{-}-\mathbf{2}^{-}\right)+n_{7} \mathbf{7}^{+}\left(\mathbf{1}^{-}-1+\mathbf{0}^{+}\right)\right] I_{a}=0,
$$

which is obtained by applying $\partial_{1} \cdot v$ to the integrand of (3.1); the case $n_{0}>0, n_{8} \neq 1$ is symmetric. When $n_{0}>0$ and $n_{6} \neq 1$, we can lower $n_{0}$ by

$$
\begin{aligned}
{[2[3 d+} & \left.n_{0}-n_{1}-n_{2}-2\left(n_{3}+n_{4}+n_{5}+n_{6}+n_{7}+n_{8}\right)\right]+ \\
& \left.+n_{3} \mathbf{3}^{+}\left(\mathbf{1}^{-}-1\right)+n_{4} \mathbf{4}^{+}\left(\mathbf{2}^{-}-1\right)-n_{6} \mathbf{6}^{+} \mathbf{0}^{+}-2 n_{0} \mathbf{0}^{-}\right] I_{a}=0
\end{aligned}
$$

which is the $\left(\partial_{1}+\partial_{2}-\partial_{3}\right) \cdot v$ relation simplified using the homogeneity relation

$$
\left[3 d+n_{0}-n_{1}-n_{2}-2\left(n_{3}+n_{4}+n_{5}+n_{6}+n_{7}+n_{8}\right)+n_{1} \mathbf{1}^{+}+n_{2} \mathbf{2}^{+}\right] I_{a}=0 .
$$

When $n_{0}>0$ and $n_{1} \neq 1$, we can lower $n_{0}$ by

$$
\begin{aligned}
{\left[d-n_{1}\right.} & -n_{3}-n_{5}-2 n_{7}+n_{1} \mathbf{1}^{+}\left(1-\mathbf{0}^{+}\right)+ \\
& \left.+n_{3} \mathbf{3}^{+}\left(\mathbf{6}^{-}-\mathbf{7}^{-}\right)+n_{5} \mathbf{5}^{+}\left(\mathbf{8}^{-}-\mathbf{7}^{-}\right)\right] I_{a}=0
\end{aligned}
$$

which is obtained by applying $\partial_{1} \cdot\left(k_{1}+k_{3}\right)$ to the integrand of (3.1); the case $n_{0}>0$, $n_{2} \neq 1$ is symmetric. We are left with $n_{0}>0, n_{1}=n_{2}=n_{6}=n_{7}=n_{8}=1$; we use $\partial_{3} \cdot\left(k_{3}+k_{1}\right)$ relation

$$
\begin{aligned}
& {\left[d+n_{0}-n_{6}-n_{8}-2 n_{7}+n_{0} \mathbf{0}^{-}\left(\mathbf{1}^{-}-1\right)+\right.} \\
& \left.+n_{6} \mathbf{6}^{+}\left(\mathbf{3}^{-}-\mathbf{7}^{-}\right)+n_{8} \mathbf{8}^{+}\left(\mathbf{5}^{-}-\mathbf{7}^{-}\right)\right] I_{a}=0
\end{aligned}
$$

to lower $n_{0}$ or raise $n_{6}$ or $n_{8}$, and apply the method described above again.

Now we shall discuss the integral $I_{a}\left(n_{1}, n_{2}, n_{3}, n_{4}, n_{5}, n_{6}, n_{7}, n_{8}\right)$ without numerator $\left(n_{0}=0\right)$. Applying $\partial_{3} \cdot k_{3}, \partial_{1} \cdot k_{1}, \partial_{1} \cdot\left(k_{1}-k_{2}\right),\left(\partial_{1}+\partial_{2}-\partial_{3}\right) \cdot k_{1}$ to the integrand of (3.1), we obtain the recurrence relations

$$
\begin{gathered}
{\left[d-n_{7}-n_{8}-2 n_{6}+n_{7} \mathbf{7}^{+}\left(\mathbf{3}^{-}-\mathbf{6}^{-}\right)+n_{8} \mathbf{8}^{+}\left(\mathbf{4}^{-}-\mathbf{6}^{-}\right)\right] I_{a}=0} \\
{\left[d-n_{1}-n_{5}-n_{7}-2 n_{3}+n_{1} \mathbf{1}^{+}+\right.} \\
\left.+n_{5} \mathbf{5}^{+}\left(\mathbf{4}^{-}-\mathbf{3}^{-}\right)+n_{7} \mathbf{7}^{+}\left(\mathbf{6}^{-}-\mathbf{3}^{-}\right)\right] I_{a}=0 \\
{\left[d-n_{1}-n_{3}-n_{7}-2 n_{5}+n_{1} \mathbf{1}^{+} \mathbf{2}^{-}+\right.} \\
\left.+n_{3} \mathbf{3}^{+}\left(\mathbf{4}^{-}-\mathbf{5}^{-}\right)+n_{7} \mathbf{7}^{+}\left(\mathbf{8}^{-}-\mathbf{5}^{-}\right)\right] I_{a}=0 \\
{\left[2\left(d-n_{5}-n_{7}-n_{8}\right)-n_{2}-n_{4}-n_{6}+n_{2} \mathbf{2}^{+} \mathbf{1}^{-}+\right.} \\
\left.+n_{4} \mathbf{4}^{+}\left(\mathbf{3}^{-}-\mathbf{5}^{-}\right)+n_{6} \mathbf{6}^{+}\left(\mathbf{3}^{-}-\mathbf{7}^{-}\right)\right] I_{a}=0
\end{gathered}
$$

where the last relation was simplified using (3.4). 
The cases $n_{1}=0, n_{2}=0, n_{6}=0, n_{7}=0, n_{8}=0$ are trivial. When $n_{1}<0$, it can be raised by

$$
\begin{aligned}
& {\left[3 d-n_{1}-n_{2}-2\left(n_{3}+n_{4}+n_{5}+n_{6}+n_{7}+n_{8}\right)\right] I_{a}=} \\
& \quad=\left[d-n_{1}-n_{2}-n_{4}-n_{8}-2 n_{5}+n_{4} \mathbf{4}^{+}\left(\mathbf{3}^{-}-\mathbf{5}^{-}\right)+n_{8} \mathbf{8}^{+}\left(\mathbf{7}^{-}-\mathbf{5}^{-}\right)\right] \mathbf{1}^{+} I_{a}
\end{aligned}
$$

which is the difference of (3.4) and $\mathbf{1}^{+}$shifted version of the relation symmetric to (3.9); the case $n_{2}<0$ is symmetric. When $n_{6}<0$ and $n_{7} \neq 1$, we can raise $n_{6}$ by (3.8) (the case $n_{8} \neq 1$ is symmetric); when $n_{6}<0$ and $n_{7}=n_{8}=1$, we can raise $n_{6}$ or $n_{8}$ by (3.6). When $n_{7}<0$ and $n_{8} \neq 1$, we can raise $n_{7}$ by the relation symmetric to (3.9); when $n_{7}<0$ and $n_{6} \neq 1$, we can raise $n_{7}$ by (3.10); when $n_{7}<0$ and $n_{6}=n_{8}=1$, we can raise $n_{7}$ or $n_{6}$ by the relation symmetric to (3.6). The case $n_{8}<0$ is symmetric. The case $n_{3}=0$ (figure 12 $a$, $\left.J_{a}\left(n_{1}, n_{2}, n_{4}, n_{5}, n_{6}, n_{7}, n_{8}\right)\right)$ will be considered later in this section; the case $n_{4}=0$ is symmetric. When $n_{3}<0$ and $n_{6} \neq 1$, we can raise $n_{3}$ by (3.6); when $n_{3}<0$ and $n_{7} \neq 1$, we can raise $n_{3}$ by (3.7); when $n_{3}<0$ and $n_{6}=n_{7}=1$, we can raise $n_{6}$ or $n_{7}$ by the relation symmetric to (3.6). The case $n_{4}<0$ is symmetric. The case $n_{5}=0$ (figure $\left[12 b, J_{b}\left(n_{1}, n_{2}, n_{3}, n_{4}, n_{6}, n_{7}, n_{8}\right)\right.$ ) will be considered later in this section. When $n_{5}<0$ and $n_{8} \neq 1$, we can raise $n_{5}$ by (3.6) (the case $n_{7} \neq 1$ is symmetric); when $n_{5}<0$ and $n_{7}=n_{8}=1$, we can raise $n_{7}$ or $n_{8}$ by (3.7). When all the indices are positive, we can use (3.7) to kill one of the lines $3,4,6$; or we can use (3.6) to kill one of the lines $3,5,7$; or we can use the symmetric relation to kill one of the lines 4, 5, 8; or we can use (3.10) to kill one of the lines $1,3,5,7$; or we can use the symmetric relation to kill one of the lines $2,4,5,8$.

Now we consider $J_{a}\left(n_{1}, n_{2}, n_{4}, n_{5}, n_{6}, n_{7}, n_{8}\right)=I_{a}\left(n_{1}, n_{2}, 0, n_{3}, n_{4}, n_{5}, n_{6}, n_{7}, n_{8}\right)$ (figure $12 a$ ). This integral vanishes when the indices of the following groups of lines are non-positive: $12,67,68,78,57,15,17,46,485,425,248$, or 258 . If any index is zero, the integral becomes trivial. If $n_{1}<0, n_{2}<0, n_{6}<0, n_{7}<0$, or $n_{8}<0$, we just consider $J_{a}$ as $I_{a}$ with $n_{3}=0$ and proceed as usual; the integral reduces to trivial ones not including $J_{a}$. When $n_{4}>0$, we can kill one of the lines 2, 4, 8 using the relation symmetric to (3.10). When $n_{5}>0$, we can kill one of the lines $2,5,8$ using (3.9). When both $n_{4}$ and $n_{5}$ are negative, we proceed as follows. If $n_{6} \neq 1$, $n_{4}$ can be raised by the relation symmetric to (3.10); if $n_{7} \neq 1, n_{5}$ can be raised by (3.9); $n_{2}$ can be lowered down to 1 using (3.4). The remaining integral can be calculated by a repeated use of (2.13):

$$
\begin{aligned}
J_{a}\left(n_{1}, 1,-\mid\right. & \left.n_{4}|,-| n_{5} \mid, 1,1, n_{8}\right)= \\
= & \sum_{\substack{l_{1}, l_{2}, l_{3}, m\\
}} \frac{(-1)^{l_{1}+l_{3}}\left|n_{4}\right| !\left|n_{5}\right| !}{\left(\left|n_{5}\right|-l_{1}\right) ! m !\left(l_{1}-2 m\right) !\left(\left|n_{4}\right|-l_{2}\right) ! l_{3} !\left(l_{2}-l_{3}\right) !} \times \\
& \times I\left(n_{1}, 1 ; l_{1}, m\right) I\left(1, n_{8}-\left|n_{4}\right|-\left|n_{5}\right|+l_{1}+l_{2}-m ; l_{2}, 0\right) \times \\
& \times I\left(-2\left(d+\left|n_{4}\right|+\left|n_{5}\right|-n_{8}\right)+n_{1}+l_{3}+3,1\right) .
\end{aligned}
$$


Finally, we consider $J_{b}\left(n_{1}, n_{2}, n_{3}, n_{4}, n_{6}, n_{7}, n_{8}\right)=I_{a}\left(n_{1}, n_{2}, n_{3}, n_{4}, 0, n_{6}, n_{7}, n_{8}\right)$ (figure 12b). This integral is mirror-symmetric; it vanishes when the indices of the following groups of lines are non-positive: $12,67,68,78,37,48,13,17,24,28$, or 364. If any index is zero, the integral becomes trivial. If $n_{1}<0, n_{2}<0, n_{3}<0$, $n_{4}<0, n_{6}<0, n_{7}<0$, or $n_{8}<0$, we just consider $J_{b}$ as $I_{a}$ with $n_{5}=0$ and proceed as usual; the integral reduces to trivial ones not including $J_{b}$ (though, possibly, including $J_{a}$ ). When all the indices are positive, one of the lines $3,4,6$ can be killed by (3.7).

\subsection{Mercedez with three heavy-quark lines}

Now let's consider the diagram of figure 11 $b$. We define

$$
\begin{aligned}
& \int \frac{N^{n_{0}} d^{d} k_{1} d^{d} k_{2} d^{d} k_{3}}{D_{1}^{n_{1}} D_{2}^{n_{2}} D_{3}^{n_{3}} D_{4}^{n_{4}} D_{5}^{n_{5}} D_{6}^{n_{6}} D_{7}^{n_{7}} D_{8}^{n_{8}}}= \\
& =-i \pi^{3 d / 2}(-2 \omega)^{3 d+2 n_{0}-2 \sum_{i=4}^{8} n_{i}} I_{b}\left(n_{1}, n_{2}, n_{3}, n_{4}, n_{5}, n_{6}, n_{7}, n_{8} ; n_{0}\right) \text {, } \\
& D_{1}=\frac{\left(k_{1}+p\right) \cdot v}{\omega}, \quad D_{2}=\frac{\left(k_{2}+p\right) \cdot v}{\omega}, \quad D_{3}=\frac{\left(k_{3}+p\right) \cdot v}{\omega} \text {, } \\
& D_{4}=-k_{1}^{2}, \quad D_{5}=-k_{2}^{2}, \quad D_{6}=-\left(k_{1}-k_{3}\right)^{2} \text {, } \\
& D_{7}=-\left(k_{2}-k_{3}\right)^{2}, \quad D_{8}=-\left(k_{1}-k_{2}\right)^{2}, \quad N=-k_{3}^{2} \text {. }
\end{aligned}
$$

This integral is mirror-symmetric with respect to $1 \leftrightarrow 2,4 \leftrightarrow 5,6 \leftrightarrow 7$. It vanishes when the indices of the following groups of lines are non-positive: 36, 37, 67, 123, $148,168,416,486,258,278,527$, or 587.

First we are going to get rid of the numerator. When $n_{4} \neq 1$, we can lower $n_{0}$ by the $\partial_{1} \cdot\left(k_{1}-k_{3}\right)$ relation

$$
\left[d-n_{1}-n_{4}-n_{8}-2 n_{6}+n_{1} \mathbf{1}^{+} \mathbf{3}^{-}+n_{4} \mathbf{4}^{+}\left(\mathbf{0}^{+}-\mathbf{6}^{-}\right)+n_{8} \mathbf{8}^{+}\left(\mathbf{7}^{-}-\mathbf{6}^{-}\right)\right] I_{b}=0 ;
$$

the case $n_{5} \neq 0$ is symmetric. When $n_{6} \neq 1$, we can lower $n_{0}$ by the $\partial_{1} \cdot k_{1}$ relation

$$
\left[d-n_{1}-n_{6}-n_{8}-2 n_{4}+n_{1} \mathbf{1}^{+}+n_{6} \mathbf{6}^{+}\left(\mathbf{0}^{+}-\mathbf{4}^{-}\right)+n_{8} \mathbf{8}^{+}\left(\mathbf{5}^{-}-\mathbf{4}^{-}\right)\right] I_{b}=0 ;
$$

the case $n_{7} \neq 1$ is symmetric. When $n_{3} \neq 1$, we can lower $n_{0}$ or raise $n_{6}$ or $n_{7}$ by the $\partial_{3} \cdot v$ relation

$$
\left[-2 n_{3} \mathbf{3}^{+}+n_{0} \mathbf{0}^{-}\left(1-\mathbf{3}^{-}\right)+n_{6} \mathbf{6}^{+}\left(\mathbf{3}^{-}-\mathbf{1}^{-}\right)+n_{7} \mathbf{7}^{+}\left(\mathbf{3}^{-}-\mathbf{2}^{-}\right)\right] I_{b}=0 .
$$

Finally, we can lower $n_{0}$ or raise $n_{3}$ or $n_{7}$ by the $\partial_{3} \cdot\left(k_{3}-k_{1}\right)$ relation

$$
\left[d+n_{0}-n_{3}-n_{7}-2 n_{6}+n_{3} \mathbf{3}^{+} \mathbf{1}^{-}+n_{0} \mathbf{0}^{-}\left(\mathbf{6}^{-}-\mathbf{4}^{-}\right)+n_{7} \mathbf{7}^{+}\left(\mathbf{8}^{-}-\mathbf{6}^{-}\right)\right] I_{b}=0 .
$$


Now we shall discuss the integral $I_{b}\left(n_{1}, n_{2}, n_{3}, n_{4}, n_{5}, n_{6}, n_{7}, n_{8}\right)$ without numerator $\left(n_{0}=0\right)$. Applying $\partial_{1} \cdot v, \partial_{1} \cdot\left(k_{1}-k_{2}\right),\left(\partial_{1}+\partial_{2}+\partial_{3}\right) \cdot k_{1}$ to the integrand of (3.12), we obtain the recurrence relations

$$
\begin{aligned}
& {\left[-2 n_{1} \mathbf{1}^{+}+n_{4} \mathbf{4}^{+}\left(\mathbf{1}^{-}-1\right)\right.}\left.+n_{6} \mathbf{6}^{+}\left(\mathbf{1}^{-}-\mathbf{3}^{-}\right)+n_{8} \mathbf{8}^{+}\left(\mathbf{1}^{-}-\mathbf{2}^{-}\right)\right] I_{b}=0 \\
& {\left[d-n_{1}-n_{4}-n_{6}-2 n_{8}+n_{1} \mathbf{1}^{+} \mathbf{2}^{-}+\right.} \\
&\left.\quad+n_{4} \mathbf{4}^{+}\left(\mathbf{5}^{-}-\mathbf{8}^{-}\right)+n_{6} \mathbf{6}^{+}\left(\mathbf{7}^{-}-\mathbf{8}^{-}\right)\right] I_{b}=0 \\
& {\left[2\left(d-n_{6}-n_{7}-n_{8}\right)-n_{2}-n_{3}-n_{5}+\right.} \\
&\left.+\left(n_{2} \mathbf{2}^{+}+n_{3} \mathbf{3}^{+}\right) \mathbf{1}^{-}+n_{5} \mathbf{5}^{+}\left(\mathbf{4}^{-}-\mathbf{8}^{-}\right)\right] I_{b}=0
\end{aligned}
$$

where the last relation was simplified using the homogeneity relation

$$
\begin{aligned}
{\left[3 d-n_{1}-n_{2}-n_{3}-2\left(n_{4}\right.\right.} & \left.+n_{5}+n_{6}+n_{7}+n_{8}\right)+ \\
& \left.+n_{1} \mathbf{1}^{+}+n_{2} \mathbf{2}^{+}+n_{3} \mathbf{3}^{+}\right] I_{b}=0 .
\end{aligned}
$$

Using it to simplify the sum of (3.17), its symmetric and (3.15), we get

$$
\begin{aligned}
{\left[2 \left[3 d-n_{1}-n_{2}-n_{3}-\right.\right.} & \left.2\left(n_{4}+n_{5}+n_{6}+n_{7}+n_{8}\right)\right]+ \\
& \left.+n_{4} \mathbf{4}^{+}\left(\mathbf{1}^{-}-1\right)+n_{5} \mathbf{5}^{+}\left(\mathbf{2}^{-}-1\right)\right] I_{b}=0 .
\end{aligned}
$$

The cases $n_{3}=0, n_{4}=0, n_{5}=0, n_{6}=0, n_{7}=0$ are trivial. When $n_{3}<0$ and $n_{6} \neq 1$, we can raise $n_{3}$ by (3.17) (the case $n_{7} \neq 1$ is symmetric); when $n_{3}<0$ and $n_{6}=n_{7}=1$, we can raise $n_{3}$ or $n_{7}$ by (3.16). When $n_{4}<0$ and $n_{5} \neq 1$, we can raise $n_{4}$ by the relation symmetric to (3.18); when $n_{4}<0$ and $n_{5}=1$, we can raise $n_{4}$ or $n_{5}$ by (3.21). The case $n_{5}<0$ is symmetric. When $n_{6}<0$ and $n_{7} \neq 1$, we can raise $n_{6}$ by (3.16); when $n_{6}<0$ and $n_{3} \neq 1, n_{7}=1$, we can raise $n_{6}$ or $n_{7}$ by (3.15); when $n_{6}<0$ and $n_{3}=n_{7}=1$, we can raise $n_{6}$ or $n_{3}$ by the relation symmetric to (3.16). The case $n_{7}<0$ is symmetric. The case $n_{1}=0$ is $J_{a}\left(n_{3}, n_{2}, n_{5}, n_{7}, n_{4}, n_{6}, n_{8}\right)$ (figure 12a, section 3.1), $n_{2}=0$ is symmetric. When $n_{1}<0$ and $n_{8} \neq 1$, we can raise $n_{1}$ by the relation symmetric to (3.17); when $n_{1}<0$ and $n_{6} \neq 1$, we can raise $n_{1}$ by (3.15); when $n_{1}<0$ and $n_{4} \neq 1$, we can raise $n_{1}$ by (3.21); when $n_{1}<0$ and $n_{2} \neq 1$, we can raise $n_{1}$ by the relation symmetric to (3.18); when $n_{1}<0$ and $n_{3} \neq 1$, we can raise $n_{1}$ by (3.16); when $n_{1}<0$ and $n_{2}=n_{3}=n_{4}=n_{6}=n_{8}=1$, we can raise $n_{1}, n_{2}$ or $n_{3}$ by (3.20). The case $n_{2}<0$ is symmetric. The case $n_{8}=0$ (figure $12 c, J_{c}\left(n_{1}, n_{2}, n_{3}, n_{4}, n_{5}, n_{6}, n_{7}\right)$ ) will be considered later in this section. When $n_{8}<0$ and $n_{7} \neq 1$, we can raise $n_{8}$ by (3.16) (the case $n_{6} \neq 1$ is symmetric); when $n_{8}<0$ and $n_{5} \neq 1$, we can raise $n_{8}$ by (3.19) 
(the case $n_{4} \neq 1$ is symmetric); when $n_{8}<0$ and $n_{1} \neq 1$, we can raise $n_{8}, n_{4}$ or $n_{6}$ by (3.17) (the case $n_{2} \neq 1$ is symmetric); when $n_{8}<0$ and $n_{3} \neq 1$, we can raise $n_{6}$ or $n_{7}$ by (3.15); when $n_{8}<0$ and $n_{1}=n_{2}=n_{3}=n_{4}=n_{5}=n_{6}=n_{7}=1$, we can raise $n_{1}, n_{2}$ or $n_{3}$ by (3.20). When all the indices are positive, we can kill one of the lines $1,6,8$ by (3.16), or one of the lines $2,7,8$ by its mirror-symmetric relation.

Now we consider $J_{c}\left(n_{1}, n_{2}, n_{3}, n_{4}, n_{5}, n_{6}, n_{7}\right)=I_{b}\left(n_{1}, n_{2}, n_{3}, n_{4}, n_{5}, n_{6}, n_{7}, n_{8}\right)$ (figure $12 c$ ). It is mirror-symmetric; it vanishes if any two indices of $n_{4}, n_{5}, n_{6}, n_{7}$ are non-positive, or $n_{1}, n_{2}, n_{3}$ are all non-positive. If any index is zero, the integral becomes trivial. If any index is negative, we just consider $J_{c}$ as $I_{b}$ with $n_{8}=0$ and proceed as usual. Using the $\partial_{1} \cdot v$ and $\partial_{3} \cdot v$ relations

$$
\begin{gathered}
{\left[-2 n_{1} \mathbf{1}^{+}+n_{4} \mathbf{4}^{+}\left(\mathbf{1}^{-}-1\right)+n_{6} \mathbf{6}^{+}\left(\mathbf{1}^{-}-\mathbf{3}^{-}\right)\right] J_{c}=0} \\
{\left[-2 n_{3} \mathbf{3}^{+}+n_{6} \mathbf{6}^{+}\left(\mathbf{3}^{-}-\mathbf{1}^{-}\right)+n_{7} \mathbf{7}^{+}\left(\mathbf{3}^{-}-\mathbf{2}^{-}\right)\right] J_{c}=0}
\end{gathered}
$$

we can lower $n_{1}, n_{2}$ (symmetric case) and $n_{3}$ down to 1 .

We are left with $J_{c}\left(1,1,1, n_{4}, n_{5}, n_{6}, n_{7}\right)$. It is rather difficult to apply the standard techniques to this integral, because each operator $\partial_{i} \cdot k_{j}$ produces a scalar product which cannot be expressed via the denominators. For example, $\partial_{1} \cdot k_{1}$ gives the term $-\frac{n_{6}}{D_{6}} 2 k_{1} \cdot k_{3}$, and $\partial_{1} \cdot\left(k_{1}-k_{3}\right)$ gives the term $-\frac{n_{4}}{D_{4}} 2 k_{1} \cdot k_{3}$. We can cancel $2 k_{1} \cdot k_{3}$ by forming the difference

$$
\left(n_{4}-1\right) \mathbf{6}^{-} \partial_{1} \cdot k_{1}-\left(n_{6}-1\right) 4^{-} \partial_{1} \cdot\left(k_{1}-k_{3}\right)
$$

which results in

$$
\left[\left(n_{4}-1\right)\left(d-n_{1}-2 n_{4}+n_{1} \mathbf{1}^{+}\right) \mathbf{6}^{-}-\left(n_{6}-1\right)\left(d-n_{1}-2 n_{6}+n_{1} \mathbf{1}^{+} \mathbf{3}^{-}\right) \mathbf{4}^{-}\right] J_{c}=0 .
$$

Another useful combination is

$$
\left(n_{4}-1\right) \mathbf{5}^{-}\left(\partial_{1}+\partial_{2}+\partial_{3}\right) \cdot k_{1}-\left(n_{5}-1\right) 4^{-}\left(\partial_{1}+\partial_{2}+\partial_{3}\right) \cdot k_{2}
$$

(it can be simplified by the homogeneity relation). Using (3.22) and (3.23), we obtain at $n_{1}=n_{2}=n_{3}=1$

$$
\begin{gathered}
{\left[\left(n_{4}-1\right)\left[2\left(d-2 n_{4}-1\right)-n_{4} \mathbf{4}^{+}\left(1-\mathbf{1}^{-}\right)\right] \mathbf{6}^{-}-\right.} \\
\left.-2\left(n_{6}-1\right)\left(d-2 n_{6}-1+\mathbf{1}^{+} \mathbf{3}^{-}\right) \mathbf{4}^{-}+\left(n_{4}-1\right)\left(n_{6}-1\right)\left(\mathbf{1}^{-}-\mathbf{3}^{-}\right)\right] J_{c}=0, \\
{\left[\left(n_{4}-1\right)\left[2\left(d-n_{5}-n_{6}-n_{7}\right)+\left(\mathbf{2}^{+}+\mathbf{3}^{+}\right) \mathbf{1}^{-}\right] \mathbf{5}^{-}-\right.} \\
\left.-\left(n_{5}-1\right)\left[2\left(d-n_{4}-n_{6}-n_{7}\right)+\left(\mathbf{1}^{+}+\mathbf{3}^{+}\right) \mathbf{2}^{-}\right] \mathbf{4}^{-}\right] J_{c}=0 .
\end{gathered}
$$


One more useful relation is obtained by adding (3.22), its mirror-symmetric, formula (3.23) and using the homogeneity relation:

$$
\begin{aligned}
{\left[2 \left[3 d-n_{1}-n_{2}\right.\right.} & \left.-n_{3}-2\left(n_{4}+n_{5}+n_{6}+n_{7}\right)\right]+ \\
& \left.+n_{4} \mathbf{4}^{+}\left(\mathbf{1}^{-}-1\right)+n_{5} \mathbf{5}^{+}\left(\mathbf{2}^{-}-1\right)\right] J_{c}=0 .
\end{aligned}
$$

When $n_{6}>1$, we can lower it down to 1 (raising $n_{4}$ ) by (3.25); the case $n_{7}>1$ is symmetric. Finally, we can lower $n_{4}$ and $n_{5}$ down to 1 by (3.26) together with (3.27).

The integral $J_{c}(1,1,1,1,1,1,1)$ cannot be reduced to simpler ones (in contrast to the massless case [3]), and should be considered as a new basis integral. Its value is currently unknown, and its calculation is highly non-trivial.

\subsection{Ladder with three heavy-quark lines}

Now let's consider the diagram of figure [1]c. We define

$$
\begin{aligned}
& \int \frac{N^{n_{0}} d^{d} k_{1} d^{d} k_{2} d^{d} k_{3}}{D_{1}^{n_{1}} D_{2}^{n_{2}} D_{3}^{n_{3}} D_{4}^{n_{4}} D_{5}^{n_{5}} D_{6}^{n_{6}} D_{7}^{n_{7}} D_{8}^{n_{8}}}= \\
& =-i \pi^{3 d / 2}(-2 \omega)^{3 d+2 n_{0}-2 \sum_{i=4}^{8} n_{i}} I_{c}\left(n_{1}, n_{2}, n_{3}, n_{4}, n_{5}, n_{6}, n_{7}, n_{8} ; n_{0}\right) \text {, } \\
& D_{1}=\frac{\left(k_{1}+p\right) \cdot v}{\omega}, \quad D_{2}=\frac{\left(k_{2}+p\right) \cdot v}{\omega}, \quad D_{3}=\frac{\left(k_{3}+p\right) \cdot v}{\omega} \text {, } \\
& D_{4}=-k_{1}^{2}, \quad D_{5}=-k_{2}^{2}, \quad D_{6}=-\left(k_{1}-k_{3}\right)^{2} \text {, } \\
& D_{7}=-\left(k_{2}-k_{3}\right)^{2}, \quad D_{8}=-k_{3}^{2}, \quad N=2 k_{1} \cdot k_{2} \text {. }
\end{aligned}
$$

This integral is mirror-symmetric with respect to $1 \leftrightarrow 2,4 \leftrightarrow 5,6 \leftrightarrow 7$. It vanishes when the indices of the following groups of lines are non-positive: 14, 16, 46, 25, 27 , $57,132,637,368,378,687,485,487,586$.

First we are going to get rid of the numerator. When $n_{7} \neq 1$, we can lower $n_{0}$ by the $\partial_{3} \cdot\left(k_{3}-k_{1}\right)$ relation

$$
\begin{gathered}
{\left[d-n_{3}-n_{7}-n_{8}-2 n_{6}+n_{3} \mathbf{3}^{+} \mathbf{1}^{-}+\right.} \\
\left.+n_{7} \mathbf{7}^{+}\left(\mathbf{0}^{+}+\mathbf{4}^{-}+\mathbf{5}^{-}-\mathbf{6}^{-}\right)+n_{8} \mathbf{8}^{+}\left(\mathbf{4}^{-}-\mathbf{6}^{-}\right)\right] I_{c}=0
\end{gathered}
$$

the case $n_{6} \neq 0$ is symmetric. When $n_{5} \neq 1$, we can lower $n_{0}$ by

$$
\begin{aligned}
& {\left[2\left(d-n_{5}-n_{6}-n_{7}\right)+n_{0}-n_{2}-n_{3}-n_{8}+2 n_{0} \mathbf{0}^{-} \mathbf{4}^{-}+\right.} \\
& \left.\quad+\left(n_{2} \mathbf{2}^{+}+n_{3} \mathbf{3}^{+}\right) \mathbf{1}^{-}-n_{5} \mathbf{5}^{+} \mathbf{0}^{+}+n_{8} \mathbf{8}^{+}\left(\mathbf{4}^{-}-\mathbf{6}^{-}\right)\right] I_{c}=0
\end{aligned}
$$

which is the $\left(\partial_{1}+\partial_{2}+\partial_{3}\right) \cdot k_{1}$ relation simplified by the homogeneity relation. the case $n_{4} \neq 1$ is symmetric. When $n_{1} \neq 1$, we can lower $n_{0}$ or raise $n_{4}$ or $n_{6}$ by the $\partial_{1} \cdot v$ relation

$$
\left[-2 n_{1} \mathbf{1}^{+}+n_{0} \mathbf{0}^{-}\left(\mathbf{2}^{-}-1\right)+n_{4} \mathbf{4}^{+}\left(\mathbf{1}^{-}-1\right)+n_{6} \mathbf{6}^{+}\left(\mathbf{1}^{-}-\mathbf{3}^{-}\right)\right] I_{c}=0
$$


the case $n_{2} \neq 1$ is symmetric. Finally, we can raise $n_{1}$ or $n_{6}$ by the $\partial_{1} \cdot k_{1}$ relation

$$
\left[d+n_{0}-n_{1}-n_{6}-2 n_{4}+n_{1} \mathbf{1}^{+}+n_{6} \mathbf{6}^{+}\left(\mathbf{8}^{-}-\mathbf{4}^{-}\right)\right] I_{c}=0
$$

Now we shall discuss the integral $I_{c}\left(n_{1}, n_{2}, n_{3}, n_{4}, n_{5}, n_{6}, n_{7}, n_{8}\right)$ without numerator $\left(n_{0}=0\right)$. Applying $\partial_{3} \cdot v, \partial_{1} \cdot\left(k_{1}-k_{3}\right)$ to the integrand of (3.28), we obtain the recurrence relations

$$
\begin{array}{r}
{\left[-2 n_{3} \mathbf{3}^{+}+n_{6} \mathbf{6}^{+}\left(\mathbf{3}^{-}-\mathbf{1}^{-}\right)+n_{7} \mathbf{7}^{+}\left(\mathbf{3}^{-}-\mathbf{2}^{-}\right)+n_{8} \mathbf{8}^{+}\left(\mathbf{3}^{-}-1\right)\right] I_{c}=0} \\
{\left[d-n_{1}-n_{4}-2 n_{6}+n_{1} \mathbf{1}^{+} \mathbf{3}^{-}+n_{4} \mathbf{4}^{+}\left(\mathbf{8}^{-}-\mathbf{6}^{-}\right)\right] I_{c}=0}
\end{array}
$$

Homogeneity in $\omega$ gives the relation identical with 3.20 .

The cases $n_{1}=0, n_{2}=0, n_{4}=0, n_{5}=0, n_{6}=0, n_{7}=0$ are trivial. When $n_{1}<0$ and $n_{6} \neq 1$, we can raise $n_{1}$ by $(3.33)$; when $n_{1}<0$ and $n_{4} \neq 1$, we can raise $n_{1}$ using the sum of (3.31) and (3.33); when $n_{1}<0$ and $n_{4}=n_{6}=1$, we can raise $n_{1}$ or $n_{6}$ by (3.32). The case $n_{2}<0$ is symmetric. When $n_{4}<0$ and $n_{6} \neq 1$, we can raise $n_{4}$ by (3.32); when $n_{4}<0$ and $n_{1} \neq 1, n_{6}=1$, we can raise $n_{4}$ or $n_{6}$ by (3.31); when $n_{4}<0$ and $n_{1}=n_{6}=1$, we can raise $n_{4}$ or $n_{1}$ by (3.34). The case $n_{5}<0$ is symmetric. When $n_{6}<0$ and $n_{4} \neq 1$, we can raise $n_{6}$ by (3.34); when $n_{6}<0$ and $n_{1} \neq 1, n_{4}=1$, we can raise $n_{6}$ or $n_{4}$ by (3.31); when $n_{6}<0$ and $n_{1}=n_{4}=1$, we can raise $n_{6}$ or $n_{1}$ by (3.32). The case $n_{7}<0$ is symmetric. The case $n_{3}=0$ is $J_{b}\left(n_{1}, n_{2}, n_{4}, n_{5}, n_{8}, n_{6}, n_{7}\right)$ (figure $12 a$, section 3.1). When $n_{3}<0$ and $n_{6} \neq 1$, we can raise $n_{3}$ by (3.31) (the case $n_{7} \neq 1$ is symmetric); when $n_{3}<0$ and $n_{8} \neq 1$, we can raise $n_{3}$ by the relation

$$
\begin{aligned}
& {\left[2\left[3 d-n_{1}-n_{2}-n_{3}-2\left(n_{4}+n_{5}+n_{6}+n_{7}+n_{8}\right)\right]+\right.} \\
& \left.\quad+n_{4} \mathbf{4}^{+}\left(\mathbf{1}^{-}-1\right)+n_{5} \mathbf{5}^{+}\left(\mathbf{2}^{-}-1\right)+n_{8} \mathbf{8}^{+}\left(\mathbf{3}^{-}-1\right)\right] I_{c}=0
\end{aligned}
$$

which is (3.31) plus its mirror-symmetric plus (3.33) simplified by the homogeneity relation; when $n_{3}<0$ and $n_{1} \neq 1$, we can raise $n_{3}$ by (3.34) (the case $n_{2} \neq 1$ is symmetric); when $n_{3}<0$ and $n_{1}=n_{2}=n_{6}=n_{8}=1$, we can raise $n_{1}, n_{2}$ or $n_{3}$ by the homogeneity relation. The case $n_{8}=0$ is $J_{c}\left(n_{1}, n_{2}, n_{3}, n_{4}, n_{5}, n_{6}, n_{7}\right)$ (figure [12c, section 3.2). When $n_{8}<0$ and $n_{6} \neq 1$, we can raise $n_{8}$ by (3.32) (the case $n_{7} \neq 1$ is symmetric); when $n_{8}<0$ and $n_{4} \neq 1$, we can raise $n_{8}$ by (3.34) (the case $n_{5} \neq 1$ is symmetric); when $n_{8}<0$ and $n_{3} \neq 1, n_{4}=n_{6}=n_{7}=1$, we can raise $n_{8}, n_{6}$ or $n_{7}$ by (3.33); when $n_{8}<0$ and $n_{1} \neq 1, n_{3}=n_{4}=n_{5}=n_{6}=n_{7}=1$, we can raise $n_{4}$ or $n_{6}$ by (3.31) (the case $n_{2} \neq 1$ is symmetric); when $n_{8}<0$ and $n_{1}=n_{2}=n_{3}=n_{4}=n_{5}=n_{6}=n_{7}=1$, we can raise $n_{1}, n_{2}$ or $n_{3}$ the homogeneity relation. When all the indices are positive, we can kill one of the lines $3,6,8$ by (3.34), or one of the lines $3,7,8$ by its mirror-symmetric relation. 


\subsection{Non-planar diagram with three heavy-quark lines}

Now let's consider the diagram of figure 11$]$. We define

$$
\begin{array}{rlrl}
\int & \frac{N^{n_{0}} d^{d} k_{1} d^{d} k_{2} d^{d} k_{3}}{D_{1}^{n_{1}} D_{2}^{n_{2}} D_{3}^{n_{3}} D_{4}^{n_{4}} D_{5}^{n_{5}} D_{6}^{n_{6}} D_{7}^{n_{7}} D_{8}^{n_{8}}}= & \\
& =-i \pi^{3 d / 2}(-2 \omega)^{3 d+2 n_{0}-2 \sum_{i=4}^{8} n_{i}} I_{d}\left(n_{1}, n_{2}, n_{3}, n_{4}, n_{5}, n_{6}, n_{7}, n_{8} ; n_{0}\right), \\
D_{1} & =\frac{\left(k_{1}+p\right) \cdot v}{\omega}, & D_{2}=\frac{\left(k_{2}+p\right) \cdot v}{\omega}, & D_{3}=\frac{\left(k_{1}+k_{2}-k_{3}+p\right) \cdot v}{\omega}, \\
D_{4}=-k_{1}^{2}, & D_{5}=-k_{2}^{2}, & D_{6}=-\left(k_{1}-k_{3}\right)^{2}, \\
D_{7}=-\left(k_{2}-k_{3}\right)^{2}, & D_{8}=-k_{3}^{2}, & N=2 k_{1} \cdot k_{2} .
\end{array}
$$

This integral is mirror-symmetric with respect to $1 \leftrightarrow 2,4 \leftrightarrow 5,6 \leftrightarrow 7$. It vanishes

when the indices of the following groups of lines are non-positive: 46, 57, 132, 485, 487, 586, 687, 314, 136, 325, 237, 148, 178, 258, 268, 417, 526, 637, 368, 378.

When $n_{1} \leq 0, n_{2} \leq 0$ or $n_{3} \leq 0$, we use

$$
\begin{aligned}
& I_{d}\left(-\left|n_{1}\right|, n_{2}, n_{3}, n_{4}, n_{5}, n_{6}, n_{7}, n_{8} ; n_{0}\right)= \\
& =\left(\mathbf{0}^{+}+\mathbf{1}^{-}\right)^{\left|n_{1}\right|}\left(\mathbf{5}^{-}+\mathbf{6}^{-}-\mathbf{3}^{-}-\mathbf{8}^{-}\right)^{n_{0}} I_{a}\left(n_{3}, n_{2}, 0, n_{5}, n_{6}, n_{7}, n_{4}, n_{8}\right), \\
& I_{d}\left(n_{1}, n_{2},-\left|n_{3}\right|, n_{4}, n_{5}, n_{6}, n_{7}, n_{8} ; n_{0}\right)= \\
& =\left(\mathbf{0}^{+}+\mathbf{1}^{-}+\mathbf{2}^{-}-1\right)^{\left|n_{3}\right|}\left(\mathbf{5}^{-}-\mathbf{3}^{-}-\mathbf{4}^{-}\right)^{n_{0}} I_{a}\left(n_{1}, n_{2}, n_{4}, n_{5}, 0, n_{8}, n_{6}, n_{7}\right),
\end{aligned}
$$

and relation symmetric to (3.37). Applying $\partial_{2} \cdot v$ and $-\partial_{3} \cdot v$ to the integrand of (3.36), we obtain

$$
\begin{gathered}
{\left[2 n_{2} \mathbf{2}^{+}+2 n_{3} \mathbf{3}^{+}+n_{5} \mathbf{5}^{+}\left(1-\mathbf{2}^{-}\right)+n_{7} \mathbf{7}^{+}\left(\mathbf{1}^{-}-\mathbf{3}^{-}\right)\right] I_{d}=0} \\
{\left[2 n_{3} \mathbf{3}^{+}+n_{6} \mathbf{6}^{+}\left(\mathbf{2}^{-}-\mathbf{3}^{-}\right)+n_{7} \mathbf{7}^{+}\left(\mathbf{1}^{-}-\mathbf{3}^{-}\right)+\right.} \\
\left.+n_{8} \mathbf{8}^{+}\left(\mathbf{1}^{-}+\mathbf{2}^{-}-\mathbf{3}^{-}-1\right)\right] I_{d}=0 .
\end{gathered}
$$

When $n_{1}>1$, we can lower it by

$$
\begin{aligned}
& {\left[2\left[3 d-n_{1}-n_{2}-n_{3}-2\left(n_{4}+n_{5}+n_{6}+n_{7}+n_{8}-n_{0}\right)\right]+\right.} \\
& \left.+n_{0} \mathbf{0}^{-}\left(\mathbf{1}^{-}-1\right)+2 n_{1} \mathbf{1}^{+}+n_{5} \mathbf{5}^{+}\left(\mathbf{2}^{-}-1\right)+n_{7} \mathbf{7}^{+}\left(\mathbf{3}^{-}-\mathbf{1}^{-}\right)\right] I_{d}=0
\end{aligned}
$$

which is twice the homogeneity relation

$$
\begin{aligned}
{\left[3 d-n_{1}-n_{2}-n_{3}-2\left(n_{4}+n_{5}\right.\right.} & \left.+n_{6}+n_{7}+n_{8}-n_{0}\right)+ \\
& \left.+n_{1} \mathbf{1}^{+}+n_{2} \mathbf{2}^{+}+n_{3} \mathbf{3}^{+}\right] I_{d}=0
\end{aligned}
$$

minus (3.39); the case $n_{2}>1$ is symmetric. When $n_{3}>1$, we can lower it by (3.40). 
We are left with $I_{d}\left(1,1,1, n_{4}, n_{5}, n_{6}, n_{7}, n_{8} ; n_{0}\right)$, and now are going to get rid of the numerator. Applying $\partial_{3} \cdot\left(k_{3}-k_{2}\right)$ and $\partial_{1} \cdot\left(k_{1}-k_{3}\right)$ to the integrand of (3.36), we obtain

$$
\begin{gathered}
{\left[d-n_{3}-n_{6}-n_{8}-2 n_{7}+n_{3} \mathbf{3}^{+} \mathbf{1}^{-}+\right.} \\
\left.+n_{6} \mathbf{6}^{+}\left(\mathbf{4}^{-}+\mathbf{5}^{-}-\mathbf{7}^{-}+\mathbf{0}^{+}\right)+n_{8} \mathbf{8}^{+}\left(\mathbf{5}^{-}-\mathbf{7}^{-}\right)\right] I_{d}=0 \\
{\left[d+n_{0}-n_{3}-n_{4}-2 n_{6}+n_{0} \mathbf{0}^{-}\left(\mathbf{5}^{-}-\mathbf{7}^{-}+\mathbf{8}^{-}\right)+\right.} \\
\left.+n_{1} \mathbf{1}^{+}\left(\mathbf{2}^{-}-\mathbf{3}^{-}\right)+n_{3} \mathbf{3}^{+} \mathbf{2}^{-}+n_{4} \mathbf{4}^{+}\left(\mathbf{8}^{-}-\mathbf{6}^{-}\right)\right] I_{d}=0 .
\end{gathered}
$$

When $n_{4} \neq 1$, we can lower $n_{0}$ by

$$
\begin{gathered}
{\left[2\left(d-n_{4}-n_{6}-n_{7}\right)+n_{0}-n_{1}-n_{2}-n_{3}-n_{8}+1-\right.} \\
-\left[3 d-n_{1}-n_{2}-n_{3}+1-2\left(n_{4}+n_{5}+n_{6}+n_{7}+n_{8}-n_{0}\right)\right] \mathbf{2}^{-}+ \\
\left.+2 n_{0} \mathbf{0}^{-} \mathbf{5}^{-}-n_{4} \mathbf{4}^{+} \mathbf{0}^{+}+n_{8} \mathbf{8}^{+}\left(\mathbf{5}^{-}-\mathbf{7}^{-}\right)\right] I_{d}=0,
\end{gathered}
$$

which is the $-\left(\partial_{1}+\partial_{2}+\partial_{3}\right) \cdot k_{2}$ relation plus (3.42) minus $2^{-}$shifted (3.42); the case $n_{5} \neq 1$ is symmetric. When $n_{6} \neq 1$, we can lower $n_{0}$ by (3.43); the case $n_{7} \neq 1$ is symmetric. Finally, when $n_{4}=n_{5}=n_{6}=n_{7}=1$, we can lower $n_{0}$ or raise $n_{4}$ by (3.44).

Now we shall discuss the integral $I_{d}\left(n_{1}, n_{2}, n_{3}, n_{4}, n_{5}, n_{6}, n_{7}, n_{8}\right)$ without numerator $\left(n_{0}=0\right)$. Applying $\partial_{1} \cdot k_{1}, \partial_{3} \cdot k_{3},\left(\partial_{2}+\partial_{3}\right) \cdot k_{3}$ to the integrand of (3.36), we obtain

$$
\begin{gathered}
{\left[d-n_{1}-n_{6}-2 n_{4}+n_{1} \mathbf{1}^{+}+n_{3} \mathbf{3}^{+}\left(1-\mathbf{1}^{-}\right)+n_{6} \mathbf{6}^{+}\left(\mathbf{8}^{-}-\mathbf{4}^{-}\right)\right] I_{d}=0} \\
{\left[d-n_{3}-n_{6}-n_{7}-2 n_{8}+n_{3} \mathbf{3}^{+}\left(\mathbf{1}^{-}+\mathbf{2}^{-}-1\right)+\right.} \\
\left.+n_{6} \mathbf{6}^{+}\left(\mathbf{4}^{-}-\mathbf{8}^{-}\right)+n_{7} \mathbf{7}^{+}\left(\mathbf{5}^{-}-\mathbf{8}^{-}\right)\right] I_{d}=0 \\
{\left[d-n_{2}-n_{5}-n_{6}-2 n_{8}+n_{2} \mathbf{2}^{+}\left(1+\mathbf{3}^{-}-\mathbf{1}^{-}\right)+\right.} \\
\left.+n_{5} \mathbf{5}^{+}\left(\mathbf{7}^{-}-\mathbf{8}^{-}\right)+n_{6} \mathbf{6}^{+}\left(\mathbf{4}^{-}-\mathbf{8}^{-}\right)\right] I_{d}=0 .
\end{gathered}
$$

Some other useful relations are

$$
\begin{aligned}
& {\left[2 n_{2} \mathbf{2}^{+}+n_{5} \mathbf{5}^{+}\left(1-\mathbf{2}^{-}\right)+n_{6} \mathbf{6}^{+}\left(\mathbf{3}^{-}-\mathbf{2}^{-}\right)+n_{8} \mathbf{8}^{+}\left(1+\mathbf{3}^{-}-\mathbf{1}^{-}-\mathbf{2}^{-}\right)\right] I_{d}=0,} \\
& {\left[2\left(d-n_{5}-n_{7}-n_{8}\right)-n_{2}-n_{3}-n_{6}+n_{2} \mathbf{2}^{+}+n_{3} \mathbf{3}^{+} \mathbf{1}^{-}+n_{6} \mathbf{6}^{+}\left(\mathbf{4}^{-}-\mathbf{8}^{-}\right)\right] I_{d}=0,}
\end{aligned}
$$

the differences of (3.39) and (3.40), and of (3.42) and (3.46).

The case $n_{1}=0$ is $J_{a}\left(n_{3}, n_{2}, n_{5}, n_{6}, n_{7}, n_{4}, n_{8}\right)$ (figure [2] $a$, section 3.1; $n_{2}=0$ is symmetric); the case $n_{3}=0$ is $J_{b}\left(n_{1}, n_{2}, n_{4}, n_{5}, n_{8}, n_{6}, n_{7}\right)$ (figure [12 $b$, section 3.1]); 
the case $n_{8}=0$ is $J_{c}\left(n_{1}, n_{2}, n_{3}, n_{4}, n_{5}, n_{7}, n_{6}\right)$ (figure $12 c$, section 3.2). The cases $n_{6}=0\left(J_{d}\left(n_{1}, n_{3}, n_{2}, n_{4}, n_{7}, n_{5}, n_{8}\right)\right.$, figure $12 d ; n_{7}=0$ is symmetric) and $n_{4}=0$ $\left(J_{e}\left(n_{1}, n_{3}, n_{2}, n_{6}, n_{8}, n_{5}, n_{7}\right)\right.$, figure $12 e ; n_{5}=0$ is symmetric) will be discussed later in this section. When $n_{2}<0$, it can be raised using

$$
\begin{aligned}
& {\left[3 d-n_{1}-n_{2}-n_{3}-2\left(n_{4}+n_{5}+n_{6}+n_{7}+n_{8}\right)\right] I_{d}=} \\
& =\left[d-n_{2}-n_{3}-n_{4}-2 n_{6}-n_{1} \mathbf{1}^{+} \mathbf{3}^{-}+n_{4} \mathbf{4}^{+}\left(\mathbf{8}^{-}-\mathbf{6}^{-}\right)\right] \mathbf{2}^{+} I_{d},
\end{aligned}
$$

which is (3.42) minus $2^{+}$shifted (3.44); the case $n_{1}<0$ is symmetric. When $n_{3}<0$ and $n_{1} \neq 1$, we can raise $n_{3}$ by (3.44) $\left(n_{2} \neq 1\right.$ is symmetric); when $n_{3}<0$ and $n_{7} \neq 1$, we can raise $n_{3}$ by (3.39) $\left(n_{6} \neq 1\right.$ is symmetric); when $n_{3}<0$ and $n_{8} \neq 1$, we can raise $n_{3}$ using

$$
\begin{gathered}
{\left[2\left[3 d-n_{1}-n_{2}-n_{3}-2\left(n_{4}+n_{5}+n_{6}+n_{7}+n_{8}\right)\right]+\right.} \\
\left.+n_{4} \mathbf{4}^{+}\left(\mathbf{1}^{-}-1\right)+n_{5} \mathbf{5}^{+}\left(\mathbf{2}^{-}-1\right)+n_{8} \mathbf{8}^{+}\left(\mathbf{1}^{-}+\mathbf{2}^{-}-\mathbf{3}^{-}-1\right)\right] I_{d}=0,
\end{gathered}
$$

which is (3.39) plus its symmetric minus (3.40) simplified by (3.42); when $n_{3}<0$ and $n_{1}=n_{2}=n_{6}=n_{7}=n_{8}=1$, we can raise $n_{3}, n_{1}$ or $n_{2}$ by (3.42). When $n_{1}>1$, it can be lowered by (3.41); the case $n_{2}>1$ is symmetric. When $n_{3}>1$, it can be lowered by (3.40).

We are left with $I_{d}\left(1,1,1, n_{4}, n_{5}, n_{6}, n_{7}, n_{8}\right)$. When $n_{4}<0$, it can be raised by (3.44); the case $n_{5}<0$ is symmetric. When $n_{6}<0$ and $n_{4} \neq 1$, we can raise $n_{6}$ by (3.44); when $n_{6}<0$ and $n_{4}=1$, we can raise $n_{6}$ or $n_{4}$ using

$$
\begin{aligned}
{\left[2\left(d-n_{1}-n_{6}-2 n_{4}\right)\right.} & -2 n_{3} \mathbf{3}^{+} \mathbf{1}^{-}+n_{4} \mathbf{4}^{+}\left(\mathbf{1}^{-}-1\right)+ \\
+ & \left.n_{6} \mathbf{6}^{+}\left[2\left(\mathbf{8}^{-}-\mathbf{4}^{-}\right)+\mathbf{3}^{-}-\mathbf{2}^{-}\right]\right] I_{d}=0,
\end{aligned}
$$

which is twice (3.46) minus the relation symmetric to (3.39). The case $n_{7}<0$ is symmetric. When $n_{8}<0$ and $n_{4} \neq 1$, we can raise $n_{8}$ by (3.44) ( $n_{5} \neq 1$ is symmetric); when $n_{8}<0$ and $n_{6} \neq 1$, we can raise $n_{8}$ by (3.53) $\left(n_{7} \neq 1\right.$ is symmetric); when $n_{8}<0$ and $n_{1}=n_{2}=n_{3}=n_{4}=n_{5}=n_{6}=n_{7}=1$, we can raise $n_{8}, n_{4}$ or $n_{5}$ by (3.52). When all the indices are positive, we can kill the line 6 or 8 using (3.44).

Thus, the non-planar diagram reduces to planar ones, in contrast to the massless case where such a reduction is impossible [3, 22].

Now we consider $J_{d}\left(n_{1}, n_{2}, n_{3}, n_{4}, n_{5}, n_{7}, n_{8}\right)=I_{d}\left(n_{1}, n_{2}, n_{3}, n_{4}, n_{5}, 0, n_{7}, n_{8}\right)$ (figure $12 d)$. This integral vanishes when indices of the following groups of lines are non-positive: $4,13,23,57,58,78,37,38,25,28$. It becomes trivial if any of the indices is zero. Applying $\left(\partial_{2}+\partial_{3}\right) \cdot k_{2}$ and $\partial_{3} \cdot\left(k_{3}-k_{2}\right)$ to the integrand of (3.36), we obtain

$$
\begin{array}{r}
{\left[d-n_{2}-n_{8}-2 n_{5}+n_{2} \mathbf{2}^{+}+n_{8} \mathbf{8}^{+}\left(\mathbf{7}^{-}-\mathbf{5}^{-}\right)\right] J_{d}=0} \\
{\left[d-n_{3}-n_{8}-2 n_{7}+n_{3} \mathbf{3}^{+} \mathbf{1}^{-}+n_{8} \mathbf{8}^{+}\left(\mathbf{5}^{-}-\mathbf{7}^{-}\right)\right] J_{d}=0}
\end{array}
$$


When $n_{3}<0$ and $n_{2} \neq 1$, we can raise $n_{3}$ by (3.48); when $n_{3}<0$ and $n_{7} \neq 1$, we can raise $n_{3}$ by (3.41); when $n_{3}<0$ and $n_{8} \neq 1$, we can raise $n_{3}$ by (3.49); when $n_{3}<0$ and $n_{2}=n_{7}=n_{8}=1$, we can raise $n_{3}$ or $n_{2}$ by (3.50). When $n_{2}<0$ and $n_{3} \neq 1$, we can raise $n_{2}$ by (3.47); when $n_{2}<0$ and $n_{5} \neq 1$, we can raise $n_{2}$ by (3.41); when $n_{2}<0$ and $n_{8} \neq 1$, we can raise $n_{2}$ by (3.40); when $n_{2}<0$ and $n_{3}=n_{5}=n_{8}=1$, we can raise $n_{2}$ or $n_{3}$ by (3.50). When $n_{8}<0$ and $n_{7} \neq 1$, we can raise $n_{8}$ by (3.47); when $n_{8}<0$ and $n_{5} \neq 1$, we can raise $n_{8}$ by (3.48); when $n_{8}<0$ and $n_{3} \neq 1, n_{5}=n_{7}=1$, we can raise $n_{8}$ or $n_{7}$ by $(3.40)$; when $n_{8}<0$ and $n_{2} \neq 1, n_{3}=n_{5}=n_{7}=1$, we can raise $n_{8}$ or $n_{5}$ by (3.49); when $n_{8}<0$ and $n_{2}=n_{3}=n_{5}=n_{7}=1$, we can raise $n_{8}$ or $n_{2}$ by (3.54). When $n_{1}<0$ and $n_{3} \neq 1$, we can raise $n_{1}$ by (3.50); when $n_{1}<0$ and $n_{4} \neq 1$, we can raise $n_{1}$ using the relation symmetric to (3.39); when $n_{1}<0$ and $n_{2} \neq 1$, we can raise $n_{1}$ by (3.48); when $n_{1}<0$ and $n_{7} \neq 1$, we can raise $n_{1}$ by (3.41); when $n_{1}<0$ and $n_{8} \neq 1$, we can raise $n_{1}$ by (3.49); when $n_{1}<0$ and $n_{2}=n_{3}=n_{4}=n_{7}=n_{8}=1$, we can raise $n_{1}, n_{2}$ or $n_{3}$ by (3.42). When $n_{5}<0$ and $n_{8} \neq 1$, we can raise $n_{5}$ by (3.55); when $n_{5}<0$ and $n_{7} \neq 1$, we can raise $n_{5}$ by (3.47); when $n_{5}<0$ and $n_{2} \neq 1$, we can raise $n_{5}$ or $n_{8}$ by (3.49); when $n_{5}<0$ and $n_{2}=n_{7}=n_{8}=1$, we can raise $n_{5}$ or $n_{2}$ by (3.48). When $n_{7}<0$ and $n_{8} \neq 1$, we can raise $n_{7}$ by (3.55); when $n_{7}<0$ and $n_{5} \neq 1$, we can raise $n_{7}$ by (3.48); when $n_{7}<0$ and $n_{3} \neq 1, n_{5}=n_{8}=1$, we can raise $n_{7}$ or $n_{8}$ by (3.40); when $n_{7}<0$ and $n_{1}=n_{3}=n_{5}=1$, we can raise $n_{7}$ or $n_{3}$ by (3.47). When all the indices are positive, we can kill one of the lines $1,5,7$ by (3.55).

Finally, we consider $J_{e}\left(n_{1}, n_{2}, n_{3}, n_{5}, n_{6}, n_{7}, n_{8}\right)=I_{d}\left(n_{1}, n_{2}, n_{3}, 0, n_{5}, n_{6}, n_{7}, n_{8}\right)$ (figure $12 e$ ). This integral vanishes when indices of the following groups of lines are non-positive: $6,13,18,17,58,78,57,325,237$. It becomes trivial if any of the indices is zero. Applying $\left(\partial_{1}+\partial_{3}\right) \cdot\left(k_{3}-k_{2}\right)$ to the integrand of (3.36), we obtain

$$
\left[d-n_{1}-n_{8}-2 n_{7}+n_{1} \mathbf{1}^{+} \mathbf{3}^{-}+n_{8} \mathbf{8}^{+}\left(\mathbf{5}^{-}-\mathbf{7}^{-}\right)\right] J_{e}=0
$$

When $n_{1}<0$, it can be raised using the relation symmetric to (3.51). When $n_{2}<0$, it can be raised by (3.51). When $n_{3}<0$ and $n_{1} \neq 1$, we can raise $n_{3}$ by (3.44); when $n_{3}<0$ and $n_{6} \neq 1$, we can raise $n_{3}$ using the relation symmetric to (3.39); when $n_{3}<0$ and $n_{2} \neq 1$, we can raise $n_{3}$ using the relation symmetric to (3.44); when $n_{3}<0$ and $n_{7} \neq 1$, we can raise $n_{3}$ by (3.39); when $n_{3}<0$ and $n_{8} \neq 1$, we can raise $n_{3}$ by (3.52); when $n_{3}<0$ and $n_{1}=n_{2}=n_{6}=n_{7}=n_{8}=1$, we can raise $n_{3}, n_{1}$ or $n_{2}$ by (3.42). When $n_{8}<0$ and $n_{7} \neq 1$, we can raise $n_{8}$ using the relation symmetric to (3.48); when $n_{8}<0$ and $n_{5} \neq 1$, we can raise $n_{8}$ using the relation symmetric to (3.44); when $n_{8}<0$ and $n_{1} \neq 1, n_{5}=n_{7}=1$, we can raise $n_{8}$ or $n_{7}$ using the relation symmetric to (3.49); when $n_{8}<0$ and $n_{1}=n_{5}=n_{7}=1$, we can raise $n_{8}$ or $n_{1}$ by (3.56). When $n_{5}<0$ and $n_{8} \neq 1$, we can raise $n_{5}$ by (3.56); when $n_{5}<0$ and $n_{7} \neq 1$, we can raise $n_{5}$ using the relation symmetric to (3.50); when $n_{5}<0$ and $n_{1} \neq 1$, we can raise $n_{8}$ or $n_{7}$ using the relation symmetric to (3.49); when $n_{5}<0$ 
and $n_{1}=n_{7}=n_{8}=1$, we can raise $n_{5}, n_{7}$ or $n_{1}$ by (3.41). When $n_{7}<0$ and $n_{8} \neq 1$, we can raise $n_{7}$ by (3.56); when $n_{7}<0$ and $n_{5} \neq 1$, we can raise $n_{7}$ using the relation symmetric to (3.44); when $n_{7}<0$ and $n_{1} \neq 1$, we can raise $n_{7}$ or $n_{8}$ using the relation symmetric to (3.49); when $n_{7}<0$ and $n_{1}=n_{5}=n_{8}=1$, we can raise $n_{7}$ or $n_{1}$ using the relation symmetric to (3.48). When all the indices are positive, we can kill one of the lines $3,5,7$ by (3.56).

\subsection{Diagrams with four heavy-quark lines}

Let's define (figure 11e)

$$
\begin{array}{rlrl}
\int \frac{N_{13}^{n_{13}} N_{23}^{n_{23}} d^{d} k_{1} d^{d} k_{2} d^{d} k_{3}}{D_{1}^{n_{1}} D_{2}^{n_{2}} D_{3}^{n_{3}} D_{4}^{n_{4}} D_{5}^{n_{5}} D_{6}^{n_{6}} D_{7}^{n_{7}} D_{8}^{n_{8}}} & & \\
& =-i \pi^{3 d / 2}(-2 \omega)^{3 d+2\left(n_{13}+n_{23}\right)-2 \sum_{i=5}^{8} n_{i}} I_{e}\left(n_{1}, n_{2}, n_{3}, n_{4}, n_{5}, n_{6}, n_{7}, n_{8} ; n_{13}, n_{23}\right), \\
D_{1} & =\frac{\left(k_{3}+p\right) \cdot v}{\omega}, & D_{2} & =\frac{\left(k_{1}+k_{3}+p\right) \cdot v}{\omega}, \\
D_{4} & =\frac{\left(k_{2}+p\right) \cdot v}{\omega}, & D_{3}=\frac{\left(k_{1}+p\right) \cdot v}{\omega} \\
D_{7} & =-k_{2}^{2}, & D_{5} & =-k_{3}^{2}, \\
N_{13} & =2 k_{1} \cdot k_{3}, & D_{8}=-k_{1}^{2} \\
D_{23} & =2 k_{2} \cdot k_{3} .
\end{array}
$$

This integral vanishes when the indices of the following groups of lines are non-

positive: $5,12,67,68,78,47,48,234,326,238$. The heavy-quark denominators are linearly dependent: $D_{1}-D_{2}+D_{3}=1$, and therefore

$$
\left[1-\mathbf{1}^{-}+\mathbf{2}^{-}-3^{-}\right] I_{e}=0
$$

The cases $n_{1} \leq 0, n_{2} \leq 0, n_{3} \leq 0$ reduce to $I_{c}, I_{d}$ :

$$
\begin{aligned}
& I_{e}\left(-\left|n_{1}\right|, n_{2}, n_{3}, n_{4}, n_{5}, n_{6}, n_{7}, n_{8} ; n_{13}, n_{23}\right)=\left(1+\mathbf{1}^{-}-\mathbf{3}^{-}\right)^{\left|n_{1}\right|} \times \\
& \times\left(\mathbf{6}^{-}-\mathbf{4}^{-}+\mathbf{8}^{-}\right)^{n_{13}}\left(\mathbf{5}^{-}-\mathbf{7}^{-}+\mathbf{8}^{-}+\mathbf{0}^{+}\right)^{n_{23}} I_{c}\left(n_{2}, n_{4}, n_{3}, 0, n_{7}, n_{5}, n_{8}, n_{6}\right), \\
& I_{e}\left(n_{1},-\left|n_{2}\right|, n_{3}, n_{4}, n_{5}, n_{6}, n_{7}, n_{8} ; n_{13}, n_{23}\right)= \\
& =\left(\mathbf{1}^{-}+\mathbf{3}^{-}-1\right)^{\left|n_{2}\right|}\left(\mathbf{6}^{-}-\mathbf{4}^{-}-\mathbf{8}^{-}\right)^{n_{13}}\left(\mathbf{0}^{+}\right)^{n_{23}} I_{c}\left(n_{1}, n_{4}, n_{3}, n_{5}, n_{7}, 0, n_{8}, n_{6}\right), \\
& I_{e}\left(n_{1}, n_{2},-\left|n_{3}\right|, n_{4}, n_{5}, n_{6}, n_{7}, n_{8} ; n_{13}, n_{23}\right)= \\
& =\left(1-\mathbf{1}^{-}+\mathbf{3}^{-}\right)^{\left|n_{3}\right|}\left(\mathbf{4}^{-}-\mathbf{6}^{-}+\mathbf{8}^{-}+\mathbf{0}^{+}\right)^{n_{13}}\left(\mathbf{0}^{+}\right)^{n_{23}} I_{d}\left(n_{1}, n_{4}, n_{2}, n_{5}, n_{7}, 0, n_{6}, n_{8}\right) .
\end{aligned}
$$

If $n_{1,2,3}$ are all positive, we can lower them by (3.58) until one of them vanish. 
Let's define (figure 11f)

$$
\begin{aligned}
& \int \frac{N_{13}^{n_{13}} N_{23}^{n_{23}} d^{d} k_{1} d^{d} k_{2} d^{d} k_{3}}{D_{1}^{n_{1}} D_{2}^{n_{2}} D_{3}^{n_{3}} D_{4}^{n_{4}} D_{5}^{n_{5}} D_{6}^{n_{6}} D_{7}^{n_{7}} D_{8}^{n_{8}}}= \\
& =-i \pi^{3 d / 2}(-2 \omega)^{3 d+2\left(n_{13}+n_{23}\right)-2 \sum_{i=5}^{8} n_{i}} I_{f}\left(n_{1}, n_{2}, n_{3}, n_{4}, n_{5}, n_{6}, n_{7}, n_{8} ; n_{13}, n_{23}\right) \text {, } \\
& D_{1}=\frac{\left(k_{1}+p\right) \cdot v}{\omega}, \quad D_{2}=\frac{\left(k_{1}+k_{3}+p\right) \cdot v}{\omega}, \quad D_{3}=\frac{\left(k_{2}+k_{3}+p\right) \cdot v}{\omega}, \\
& D_{4}=\frac{\left(k_{2}+p\right) \cdot v}{\omega}, \quad D_{5}=-k_{3}^{2}, \quad D_{6}=-k_{1}^{2}, \\
& D_{7}=-k_{2}^{2}, \quad D_{8}=-\left(k_{1}-k_{2}\right)^{2} \text {. }
\end{aligned}
$$

This integral is mirror-symmetric with respect to $1 \leftrightarrow 4,2 \leftrightarrow 3,6 \leftrightarrow 7$. It vanishes when indices of the following groups of lines are non-positive: $5,23,67,68,78,216$, $128,347,438$. The heavy-quark denominators are linearly dependent: $D_{1}-D_{2}+$ $D_{3}-D_{4}=0$, and therefore

$$
\left[\mathbf{1}^{-}-\mathbf{2}^{-}+\mathbf{3}^{-}-\mathbf{4}^{-}\right] I_{f}=0
$$

The cases $n_{1} \leq 0, n_{2} \leq 0$ reduce to $I_{c}, I_{d}$ :

$$
\begin{aligned}
& I_{f}\left(-\left|n_{1}\right|, n_{2}, n_{3}, n_{4}, n_{5}, n_{6}, n_{7}, n_{8} ; n_{13}, n_{23}\right)=\left(\mathbf{1}^{-}+\mathbf{2}^{-}-\mathbf{3}^{-}\right)^{\left|n_{1}\right|} \times \\
& \times\left(\mathbf{6}^{-}-\mathbf{4}^{-}+\mathbf{8}^{-}\right)^{n_{13}}\left(\mathbf{5}^{-}-\mathbf{7}^{-}+\mathbf{8}^{-}+\mathbf{0}^{+}\right)^{n_{23}} I_{d}\left(n_{2}, n_{4}, n_{3}, 0, n_{7}, n_{5}, n_{8}, n_{6}\right), \\
& I_{f}\left(n_{1},-\left|n_{2}\right|, n_{3}, n_{4}, n_{5}, n_{6}, n_{7}, n_{8} ; n_{13}, n_{23}\right)=\left(\mathbf{1}^{-}+\mathbf{2}^{-}-\mathbf{3}^{-}\right)^{\left|n_{2}\right|} \times \\
& \times\left(\mathbf{4}^{-}-\mathbf{6}^{-}+\mathbf{8}^{-}+\mathbf{0}^{+}\right)^{n_{13}}\left(\mathbf{7}^{-}+\mathbf{8}^{-}-\mathbf{5}^{-}\right)^{n_{23}} I_{c}\left(n_{1}, n_{3}, n_{4}, n_{6}, 0, n_{8}, n_{5}, n_{7}\right)
\end{aligned}
$$

(the cases $n_{4} \leq 0, n_{3} \leq 0$ are symmetric). If $n_{1,2,3,4}$ are all positive, we can use (3.60) to raise, say, $n_{1}$ and kill one of the lines $2,3,4$.

Let's define (figure 11 $g$ )

$$
\begin{aligned}
& \int \frac{N_{13}^{n_{13}} N_{23}^{n_{23}} d^{d} k_{1} d^{d} k_{2} d^{d} k_{3}}{D_{1}^{n_{1}} D_{2}^{n_{2}} D_{3}^{n_{3}} D_{4}^{n_{4}} D_{5}^{n_{5}} D_{6}^{n_{6}} D_{7}^{n_{7}} D_{8}^{n_{8}}}= \\
& =-i \pi^{3 d / 2}(-2 \omega)^{3 d+2\left(n_{13}+n_{23}\right)-2 \sum_{i=4}^{8} n_{i}} I_{g}\left(n_{1}, n_{2}, n_{3}, n_{4}, n_{5}, n_{6}, n_{7}, n_{8} ; n_{13}, n_{23}\right) \\
& D_{1}=\frac{\left(k_{3}+p\right) \cdot v}{\omega}, \quad D_{2}=\frac{\left(k_{1}+k_{3}+p\right) \cdot v}{\omega}, \quad D_{3}=\frac{\left(k_{2}+k_{3}+p\right) \cdot v}{\omega}, \\
& D_{4}=\frac{\left(k_{2}+p\right) \cdot v}{\omega}, \quad D_{5}=-k_{3}^{2}, \quad D_{6}=-k_{1}^{2} \text {, } \\
& D_{7}=-k_{2}^{2}, \quad D_{8}=-\left(k_{1}-k_{2}\right)^{2} \text {. }
\end{aligned}
$$

This integral vanishes when the indices of the following groups of lines are nonpositive: $5,67,68,78,26,28,123,234,347,438$. The heavy-quark denominators are linearly dependent: $D_{1}-D_{3}+D_{4}=1$, and therefore

$$
\left[1-\mathbf{1}^{-}+\mathbf{3}^{-}-4^{-}\right] I_{g}=0
$$


The cases $n_{1} \leq 0, n_{3} \leq 0, n_{4} \leq 0$ reduce to $I_{b}, I_{d}$ :

$$
\begin{aligned}
& I_{g}\left(-\left|n_{1}\right|, n_{2}, n_{3}, n_{4}, n_{5}, n_{6}, n_{7}, n_{8} ; n_{13}, n_{23}\right)=\left(1-\mathbf{2}^{-}+\mathbf{3}^{-}\right)^{\left|n_{1}\right|} \times \\
& \times\left(\mathbf{6}^{-}-\mathbf{4}^{-}+\mathbf{8}^{-}\right)^{n_{13}}\left(\mathbf{5}^{-}-\mathbf{7}^{-}+\mathbf{8}^{-}+\mathbf{0}^{+}\right)^{n_{23}} I_{d}\left(n_{2}, n_{4}, n_{3}, 0, n_{7}, n_{5}, n_{8}, n_{6}\right), \\
& I_{g}\left(n_{1}, n_{2},-\left|n_{3}\right|, n_{4}, n_{5}, n_{6}, n_{7}, n_{8} ; n_{13}, n_{23}\right)=\left(\mathbf{1}^{-}+\mathbf{2}^{-}-1\right)^{\left|n_{3}\right|} \times \\
& \times\left(\mathbf{4}^{-}-\mathbf{6}^{-}+\mathbf{8}^{-}+\mathbf{0}^{+}\right)^{n_{13}}(0+)^{n_{23}} I_{d}\left(n_{1}, n_{4}, n_{2}, n_{5}, n_{7}, 0, n_{6}, n_{8}\right), \\
& I_{g}\left(n_{1}, n_{2}, n_{3},-\left|n_{4}\right|, n_{5}, n_{6}, n_{7}, n_{8} ; n_{13}, n_{23}\right)=\left(1-\mathbf{1}^{-}+\mathbf{2}^{-}\right)^{\left|n_{4}\right|} \times \\
& \times\left(\mathbf{4}^{-}+\mathbf{6}^{-}-\mathbf{0}^{+}\right)^{n_{13}}\left(\mathbf{4}^{-}-\mathbf{5}^{-}+\mathbf{8}^{-}\right)^{n_{23}} I_{b}\left(n_{1}, n_{3}, n_{2}, n_{5}, 0, n_{6}, n_{8}, n_{7}\right) .
\end{aligned}
$$

If $n_{1,3,4}$ are all positive, we can lower them by (3.62) until one of them vanish.

\subsection{Diagrams with five heavy-quark lines}

Let's define (figure 11 $h$ )

$$
\begin{array}{rlrl}
\int \frac{N_{12}^{n_{12}} N_{13}^{n_{13}} N_{23}^{n_{23}} d^{d} k_{1} d^{d} k_{2} d^{d} k_{3}}{D_{1}^{n_{1}} D_{2}^{n_{2}} D_{3}^{n_{3}} D_{4}^{n_{4}} D_{5}^{n_{5}} D_{6}^{n_{6}} D_{7}^{n_{7}} D_{8}^{n_{8}}} & = & -i \pi^{3 d / 2}(-2 \omega)^{3 d+2\left(n_{12}+n_{13}+n_{23}\right)-2 \sum_{i=6}^{8} n_{i}} \times \\
& \times I_{h}\left(n_{1}, n_{2}, n_{3}, n_{4}, n_{5}, n_{6}, n_{7}, n_{8} ; n_{12}, n_{13}, n_{23}\right) \\
D_{1}=\frac{\left(k_{2}+p\right) \cdot v}{\omega}, & D_{2}=\frac{\left(k_{1}+k_{2}+p\right) \cdot v}{\omega}, & D_{3}=\frac{\left(k_{1}+p\right) \cdot v}{\omega}, \\
D_{4}=\frac{\left(k_{1}+k_{3}+p\right) \cdot v}{\omega}, & D_{5}=\frac{\left(k_{3}+p\right) \cdot v}{\omega}, & D_{6}=-k_{2}^{2}, \\
D_{7}=-k_{3}^{2}, & D_{8}=-k_{1}^{2}, & & \\
N_{12}=2 k_{1} \cdot k_{2}, & N_{13}=2 k_{1} \cdot k_{3}, & N_{23}=2 k_{2} \cdot k_{3} .
\end{array}
$$

This integral is mirror-symmetric with respect to $1 \leftrightarrow 5,2 \leftrightarrow 4,6 \leftrightarrow 7$. It vanishes when the indices of the following groups of lines are non-positive: $6,7,8,12,45$, 234. There are two linear relations among the heavy-quark denominators:

$$
\left[1-\mathbf{1}^{-}+\mathbf{2}^{-}-\mathbf{3}^{-}\right] I_{h}=0, \quad\left[1-\mathbf{3}^{-}+\mathbf{4}^{-}-\mathbf{5}^{-}\right] I_{h}=0
$$

The cases

$$
\begin{aligned}
& I_{h}\left(n_{1},-\left|n_{2}\right|, n_{3},-\left|n_{4}\right|, n_{5}, n_{6}, n_{7}, n_{8} ; n_{12}, n_{13}, n_{23}\right)= \\
& =K_{a}\left(n_{1}, n_{5}, n_{3}, n_{6}, n_{7}, n_{8} ;\left|n_{2}\right|,\left|n_{4}\right| ; n_{23}, n_{12}, n_{13}\right), \\
& I_{h}\left(n_{1},-\left|n_{2}\right|, n_{3}, n_{4},-\left|n_{5}\right|, n_{6}, n_{7}, n_{8} ; n_{12}, n_{13}, n_{23}\right)= \\
& =K_{b}\left(n_{1}, n_{4}, n_{3}, n_{6}, n_{7}, n_{8} ;\left|n_{2}\right|,\left|n_{5}\right|, 0,0 ; n_{12}, n_{13}, n_{23}\right), \\
& I_{h}\left(n_{1},-\left|n_{2}\right|,-\left|n_{3}\right|, n_{4}, n_{5}, n_{6}, n_{7}, n_{8} ; n_{12}, n_{13}, n_{23}\right)= \\
& =K_{b}\left(n_{1}, n_{4}, n_{5}, n_{6}, n_{8}, n_{7} ; 0,\left|n_{3}\right|, 0,\left|n_{2}\right| ; n_{23}, n_{13}, n_{12}\right), \\
& I_{h}\left(-\left|n_{1}\right|, n_{2}, n_{3}, n_{4},-\left|n_{5}\right|, n_{6}, n_{7}, n_{8} ; n_{12}, n_{13}, n_{23}\right)=
\end{aligned}
$$




$$
\begin{aligned}
& =K_{c}\left(n_{2}, n_{4}, n_{3}, n_{6}, n_{7}, n_{8} ;\left|n_{1}\right|,\left|n_{5}\right|, 0 ; n_{12}, n_{13}, n_{23}\right), \\
& I_{h}\left(-\left|n_{1}\right|, n_{2},-\left|n_{3}\right|, n_{4}, n_{5}, n_{6}, n_{7}, n_{8} ; n_{12}, n_{13}, n_{23}\right)= \\
& =K_{e}\left(n_{2}, n_{5}, n_{4}, n_{7}, n_{8}, n_{6} ;\left|n_{1}\right|, 0,\left|n_{3}\right| ; n_{12}, n_{23}, n_{13}\right),
\end{aligned}
$$

as well as the symmetric cases $n_{4} \leq 0, n_{1} \leq 0 ; n_{4} \leq 0, n_{3} \leq 0 ; n_{5} \leq 0, n_{3} \leq 0$, reduce to $I_{c}, I_{d}$, as will be discussed later in this section. When $n_{3} \leq 0$ (figure $12 f$ ), we can use

$$
\left[1^{-}-2^{-}+4^{-}-5^{-}\right] I_{h}=0
$$

to raise, say, $n_{1}$ and kill one of the lines $2,4,5$. When $n_{1,2}$ are both positive, we can use (3.64) to kill one of the lines $1,2,3$; when $n_{4,5}$ are both positive, we can kill one of the lines $3,4,5$.

Let's define (figure 11 $i$ )

$$
\begin{aligned}
\int \frac{N_{12}^{n_{12}} N_{13}^{n_{13}} N_{23}^{n_{23}} d^{d} k_{1} d^{d} k_{2} d^{d} k_{3}}{D_{1}^{n_{1}} D_{2}^{n_{2}} D_{3}^{n_{3}} D_{4}^{n_{4}} D_{5}^{n_{5}} D_{6}^{n_{6}} D_{7}^{n_{7}} D_{8}^{n_{8}}}= & -i \pi^{3 d / 2}(-2 \omega)^{3 d+2\left(n_{12}+n_{13}+n_{23}\right)-2 \sum_{i=6}^{8} n_{i} \times} \\
& \times I_{i}\left(n_{1}, n_{2}, n_{3}, n_{4}, n_{5}, n_{6}, n_{7}, n_{8} ; n_{12}, n_{13}, n_{23}\right), \\
D_{1}=\frac{\left(k_{1}+p\right) \cdot v}{\omega}, & D_{2}=\frac{\left(k_{1}+k_{2}+p\right) \cdot v}{\omega}, \\
D_{3}=\frac{\left(k_{1}+k_{2}+k_{3}+p\right) \cdot v}{\omega}, & D_{4}=\frac{\left(k_{1}+k_{3}+p\right) \cdot v}{\omega}, \quad \begin{array}{l}
D_{5}=\frac{\left(k_{2}+p\right) \cdot v}{\omega}, \\
D_{7}=-k_{1}^{2},
\end{array} D_{8}^{2},
\end{aligned}
$$

This integral vanishes when the indices of the following groups of lines are nonpositive: $6,7,8,34,123$. There are two linear relations among the heavy-quark denominators:

$$
\left[1-\mathbf{1}^{-}+\mathbf{3}^{-}-4^{-}\right] I_{i}=0, \quad\left[1-\mathbf{1}^{-}+\mathbf{2}^{-}-\mathbf{5}^{-}\right] I_{i}=0
$$

The cases

$$
\begin{aligned}
& I_{i}\left(n_{1},-\left|n_{2}\right|,-\left|n_{3}\right|, n_{4}, n_{5}, n_{6}, n_{7}, n_{8} ; n_{12}, n_{13}, n_{23}\right)= \\
& =K_{b}\left(n_{1}, n_{4}, n_{5}, n_{6}, n_{8}, n_{7} ;\left|n_{2}\right|, 0,\left|n_{3}\right|, 0 ; n_{12}, n_{23}, n_{13}\right), \\
& I_{i}\left(-\left|n_{1}\right|, n_{2},-\left|n_{3}\right|, n_{4}, n_{5}, n_{6}, n_{7}, n_{8} ; n_{12}, n_{13}, n_{23}\right)= \\
& =K_{c}\left(n_{2}, n_{4}, n_{5}, n_{6}, n_{8}, n_{7} ;\left|n_{1}\right|, 0,\left|n_{3}\right| ; n_{12}, n_{23}, n_{13}\right), \\
& I_{i}\left(-\left|n_{1}\right|,-\left|n_{2}\right|, n_{3}, n_{4}, n_{5}, n_{6}, n_{7}, n_{8} ; n_{12}, n_{13}, n_{23}\right)= \\
& =K_{d}\left(n_{3}, n_{5}, n_{4}, n_{7}, n_{6}, n_{8} ;\left|n_{1}\right|, 0,0,\left|n_{2}\right| ; n_{23}, n_{12}, n_{13}\right), \\
& I_{i}\left(-\left|n_{1}\right|, n_{2}, n_{3},-\left|n_{4}\right|, n_{5}, n_{6}, n_{7}, n_{8} ; n_{12}, n_{13}, n_{23}\right)= \\
& =K_{d}\left(n_{3}, n_{5}, n_{2}, n_{7}, n_{8}, n_{6} ; 0,\left|n_{1}\right|, 0,\left|n_{4}\right| ; n_{12}, n_{23}, n_{13}\right), \\
& I_{i}\left(n_{1}, n_{2}, n_{3},-\left|n_{4}\right|,-\left|n_{5}\right|, n_{6}, n_{7}, n_{8} ; n_{12}, n_{13}, n_{23}\right)= \\
& =K_{d}\left(n_{3}, n_{1}, n_{2}, n_{6}, n_{8}, n_{7} ; 0,\left|n_{5}\right|,\left|n_{4}\right|, 0 ; n_{12}, n_{13}, n_{23}\right),
\end{aligned}
$$




$$
\begin{aligned}
& I_{i}\left(n_{1}, n_{2},-\left|n_{3}\right|, n_{4},-\left|n_{5}\right|, n_{6}, n_{7}, n_{8} ; n_{12}, n_{13}, n_{23}\right)= \\
& =K_{e}\left(n_{4}, n_{1}, n_{2}, n_{6}, n_{7}, n_{8} ; 0,\left|n_{3}\right|,\left|n_{5}\right| ; n_{23}, n_{13}, n_{12}\right), \\
& I_{i}\left(n_{1},-\left|n_{2}\right|, n_{3},-\left|n_{4}\right|, n_{5}, n_{6}, n_{7}, n_{8} ; n_{12}, n_{13}, n_{23}\right)= \\
& =K_{f}\left(n_{1}, n_{5}, n_{3}, n_{6}, n_{7}, n_{8} ;\left|n_{2}\right|, 0,\left|n_{4}\right| ; n_{13}, n_{23}, n_{12}\right), \\
& I_{i}\left(-\left|n_{1}\right|, n_{2}, n_{3}, n_{4},-\left|n_{5}\right|, n_{6}, n_{7}, n_{8} ; n_{12}, n_{13}, n_{23}\right)= \\
& =K_{g}\left(n_{2}, n_{4}, n_{3}, n_{6}, n_{8}, n_{7} ;\left|n_{5}\right|,\left|n_{1}\right|, 0 ; n_{12}, n_{23}, n_{13}\right)
\end{aligned}
$$

reduce to $I_{c}, I_{d}$, as will be discussed later in this section. When $n_{1} \leq 0$, we can use

$$
\left[2^{-}-3^{-}+4^{-}-5^{-}\right] I_{i}=0
$$

to raise, say, $n_{2}$ and kill one of the lines $3,4,5$. When $n_{2,5}$ are both positive, we can use (3.67) to kill one of the lines $1,2,5$; when $n_{3,4}$ are both positive, we can kill one of the lines $1,3,4$.

Finally, let's define (figure 11j)

$$
\begin{aligned}
& \int \frac{N_{12}^{n_{12}} N_{13}^{n_{13}} N_{23}^{n_{23}} d^{d} k_{1} d^{d} k_{2} d^{d} k_{3}}{D_{1}^{n_{1}} D_{2}^{n_{2}} D_{3}^{n_{3}} D_{4}^{n_{4}} D_{5}^{n_{5}} D_{6}^{n_{6}} D_{7}^{n_{7}} D_{8}^{n_{8}}}=-i \pi^{3 d / 2}(-2 \omega)^{3 d+2\left(n_{12}+n_{13}+n_{23}\right)-2 \sum_{i=6}^{8}{ }^{n_{i}} \times} \\
& \times I_{j}\left(n_{1}, n_{2}, n_{3}, n_{4}, n_{5}, n_{6}, n_{7}, n_{8} ; n_{12}, n_{13}, n_{23}\right), \\
& D_{1}=\frac{\left(k_{1}+p\right) \cdot v}{\omega}, \quad D_{2}=\frac{\left(k_{1}+k_{2}+p\right) \cdot v}{\omega}, \\
& D_{3}=\frac{\left(k_{1}+k_{2}+k_{3}+p\right) \cdot v}{\omega}, \quad D_{4}=\frac{\left(k_{2}+k_{3}+p\right) \cdot v}{\omega}, \quad D_{5}=\frac{\left(k_{3}+p\right) \cdot v}{\omega}, \\
& D_{6}=-k_{1}^{2}, \quad D_{7}=-k_{2}^{2}, \omega D_{8}=-k_{3}^{2} \text {. }
\end{aligned}
$$

This integral is mirror-symmetric with respect to $1 \leftrightarrow 5,2 \leftrightarrow 4,6 \leftrightarrow 8$. It vanishes when the indices of the following groups of lines are non-positive: $6,7,8,123,234$, 345. There are two linear relations among the heavy-quark denominators:

$$
\left[1-\mathbf{1}^{-}+\mathbf{3}^{-}-4^{-}\right] I_{j}=0, \quad\left[1-\mathbf{2}^{-}+\mathbf{3}^{-}-\mathbf{5}^{-}\right] I_{j}=0 .
$$

The cases

$$
\begin{aligned}
& I_{j}\left(n_{1},-\left|n_{2}\right|,-\left|n_{3}\right|, n_{4}, n_{5}, n_{6}, n_{7}, n_{8} ; n_{12}, n_{13}, n_{23}\right)= \\
& =K_{b}\left(n_{1}, n_{4}, n_{5}, n_{6}, n_{7}, n_{8} ; 0,0,\left|n_{3}\right|,\left|n_{2}\right| ; n_{13}, n_{23}, n_{12}\right), \\
& I_{j}\left(-\left|n_{1}\right|,-\left|n_{2}\right|, n_{3}, n_{4}, n_{5}, n_{6}, n_{7}, n_{8} ; n_{12}, n_{13}, n_{23}\right)= \\
& =K_{d}\left(n_{3}, n_{5}, n_{4}, n_{8}, n_{6}, n_{7} ;\left|n_{1}\right|, 0,\left|n_{2}\right|, 0 ; n_{23}, n_{13}, n_{12}\right), \\
& I_{j}\left(-\left|n_{1}\right|, n_{2},-\left|n_{3}\right|, n_{4}, n_{5}, n_{6}, n_{7}, n_{8} ; n_{12}, n_{13}, n_{23}\right)= \\
& =K_{e}\left(n_{2}, n_{5}, n_{4}, n_{8}, n_{7}, n_{6} ;\left|n_{1}\right|,\left|n_{3}\right|, 0 ; n_{12}, n_{13}, n_{23}\right) \\
& I_{j}\left(n_{1},-\left|n_{2}\right|, n_{3},-\left|n_{4}\right|, n_{5}, n_{6}, n_{7}, n_{8} ; n_{12}, n_{13}, n_{23}\right)= \\
& =K_{f}\left(n_{1}, n_{5}, n_{3}, n_{6}, n_{8}, n_{7} ; 0,\left|n_{2}\right|,\left|n_{4}\right| ; n_{12}, n_{23}, n_{13}\right) \\
& I_{j}\left(-\left|n_{1}\right|, n_{2}, n_{3}, n_{4},-\left|n_{5}\right|, n_{6}, n_{7}, n_{8} ; n_{12}, n_{13}, n_{23}\right)= \\
& =K_{g}\left(n_{2}, n_{4}, n_{3}, n_{6}, n_{8}, n_{7} ; 0,\left|n_{1}\right|,\left|n_{5}\right| ; n_{12}, n_{23}, n_{13}\right),
\end{aligned}
$$




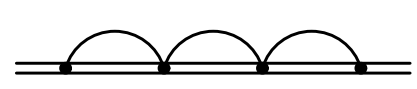

$a$

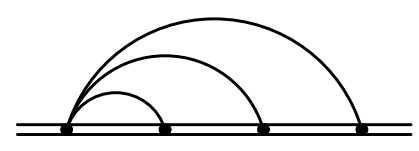

d

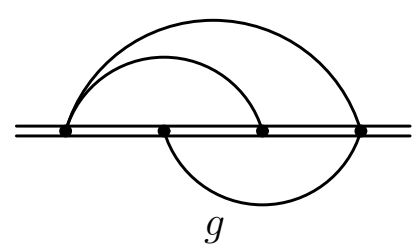

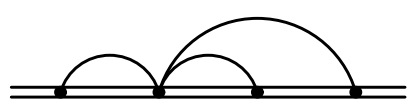

b

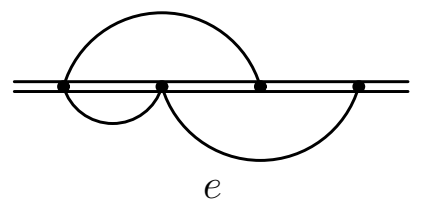

e

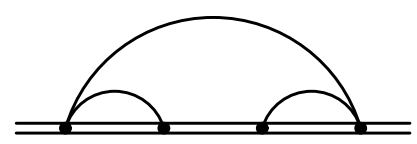

C

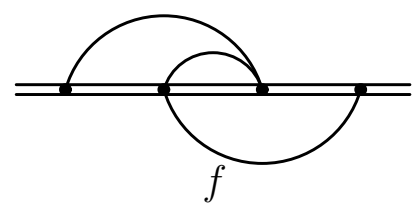

Figure 13: Reduced forms of the diagrams figure $11 h-j$

as well as the symmetric cases $n_{4} \leq 0, n_{3} \leq 0 ; n_{5} \leq 0, n_{4} \leq 0 ; n_{5} \leq 0, n_{3} \leq 0$, reduce to $I_{c}, I_{d}$, as will be discussed later in this section. When $n_{3} \leq 0$, we can use

$$
\left[\mathbf{1}^{-}-\mathbf{2}^{-}+4^{-}-5^{-}\right] I_{j}=0
$$

to raise, say, $n_{1}$ and kill one of the lines $2,4,5$. When $n_{1,4}$ are both positive, we can use (3.70) to kill one of the lines $1,3,4$; when $n_{2,5}$ are both positive, we can kill one of the lines $2,3,5$.

The reduced forms of the integrals $I_{h, i, j}$ are (figure 13)

$$
\begin{aligned}
& K_{a}\left(n_{1}, n_{2}, n_{3}, n_{4}, n_{5}, n_{8} ; n_{9}, n_{10} ; n_{45}, n_{48}, n_{58}\right)=\left(\mathbf{1}^{-}+\mathbf{3}^{-}-1\right)^{n_{9}}\left(\mathbf{2}^{-}+\mathbf{3}^{-}-1\right)^{n_{10}} \times \\
& \quad \times\left(\mathbf{0}^{+}\right)^{n_{45}}\left(\mathbf{6}^{-}-\mathbf{4}^{-}-\mathbf{8}^{-}\right)^{n_{48}}\left(\mathbf{7}^{-}-\mathbf{5}^{-}-\mathbf{8}^{-}\right)^{n_{58}} I_{c}\left(n_{1}, n_{2}, n_{3}, n_{4}, n_{5}, 0,0, n_{8}\right), \\
& K_{b}\left(n_{1}, n_{2}, n_{3}, n_{4}, n_{7}, n_{8} ; n_{9}, n_{10}, n_{11}, n_{12} ; n_{48}, n_{78}, n_{47}\right)=\left(\mathbf{1}^{-}+\mathbf{3}^{-}-1\right)^{n_{9}} \times \\
& \quad \times\left(\mathbf{2}^{-}-\mathbf{3}^{-}+1\right)^{n_{10}}\left(\mathbf{1}^{-}+\mathbf{2}^{-}-1\right)^{n_{11}}\left(\mathbf{1}^{-}+\mathbf{2}^{-}-\mathbf{3}^{-}\right)^{n_{12}}\left(\mathbf{6}^{-}-\mathbf{4}^{-}-\mathbf{8}^{-}\right)^{n_{48}} \times \\
& \quad \times\left(\mathbf{7}^{-}-\mathbf{5}^{-}+\mathbf{8}^{-}\right)^{n_{78}}\left(\mathbf{4}^{-}-\mathbf{6}^{-}+\mathbf{8}^{-}+\mathbf{0}^{+}\right)^{n_{47}} I_{c}\left(n_{1}, n_{2}, n_{3}, n_{4}, 0,0, n_{7}, n_{8}\right), \\
& K_{c}\left(n_{1}, n_{2}, n_{3}, n_{6}, n_{7}, n_{8} ; n_{9}, n_{10}, n_{11} ; n_{68}, n_{78}, n_{67}\right)=\left(\mathbf{1}^{-}-\mathbf{3}^{-}+1\right)^{n_{9}} \times \\
& \quad \times\left(\mathbf{2}^{-}-\mathbf{3}^{-}+1\right)^{n_{10}}\left(\mathbf{1}^{-}+\mathbf{2}^{-}-\mathbf{3}^{-}\right)^{n_{11}}\left(\mathbf{6}^{-}-\mathbf{4}^{-}+\mathbf{8}^{-}\right)^{n_{68}}\left(\mathbf{7}^{-}-\mathbf{5}^{-}+\mathbf{8}^{-}\right)^{n_{78}} \times \\
& \quad \times\left(\mathbf{4}^{-}+\mathbf{5}^{-}-\mathbf{6}^{-}-\mathbf{7}^{-}+\mathbf{0}^{+}\right)^{n_{67}} I_{c}\left(n_{1}, n_{2}, n_{3}, 0,0, n_{6}, n_{7}, n_{8}\right) \\
& K_{d}\left(n_{1}, n_{2}, n_{3}, n_{5}, n_{6}, n_{7} ; n_{9}, n_{10}, n_{11}, n_{12} ; n_{57}, n_{56}, n_{67}\right)=\left(\mathbf{1}^{-}-\mathbf{3}^{-}+1\right)^{n_{9}} \times \\
& \quad \times\left(\mathbf{3}^{-}-\mathbf{2}^{-}+1\right)^{n_{10}}\left(\mathbf{1}^{-}-\mathbf{2}^{-}+1\right)^{n_{11}}\left(\mathbf{1}^{-}+\mathbf{2}^{-}-\mathbf{3}^{-}\right)^{n_{12}}\left(\mathbf{5}^{-}+\mathbf{7}^{-}-\mathbf{8}^{-}\right)^{n_{57}} \times \\
& \quad \times\left(\mathbf{5}^{-}-\mathbf{7}^{-}+\mathbf{8}^{-}+\mathbf{0}^{+}\right)^{n_{56}}\left(\mathbf{6}^{-}+\mathbf{7}^{-}-\mathbf{4}^{-}-\mathbf{5}^{-}-\mathbf{0}^{+}\right)^{n_{67}} \times \\
& \quad \times I_{c}\left(n_{1}, n_{2}, n_{3}, 0, n_{5}, n_{6}, n_{7}, 0\right)
\end{aligned}
$$




$$
\begin{aligned}
& K_{e}\left(n_{1}, n_{2}, n_{3}, n_{5}, n_{6}, n_{8} ; n_{9}, n_{10}, n_{11} ; n_{68}, n_{58}, n_{56}\right)=\left(\mathbf{1}^{-}+\mathbf{2}^{-}-\mathbf{3}^{-}\right)^{n_{9}} \times \\
& \quad \times\left(\mathbf{1}^{-}+\mathbf{2}^{-}-1\right)^{n_{10}}\left(\mathbf{3}^{-}-\mathbf{2}^{-}+1\right)^{n_{11}}\left(\mathbf{6}^{-}-\mathbf{4}^{-}+\mathbf{8}^{-}\right)^{n_{68}}\left(\mathbf{7}^{-}-\mathbf{5}^{-}-\mathbf{8}^{-}\right)^{n_{58}} \times \\
& \quad \times\left(\mathbf{5}^{-}-\mathbf{7}^{-}+\mathbf{8}^{-}+\mathbf{0}^{+}\right)^{n_{56}} I_{d}\left(n_{1}, n_{2}, n_{3}, 0, n_{5}, n_{6}, 0, n_{8}\right), \\
& K_{f}\left(n_{1}, n_{2}, n_{3}, n_{4}, n_{5}, n_{8} ; n_{9}, n_{10}, n_{11} ; n_{48}, n_{58}, n_{45}\right)=\left(\mathbf{1}^{-}+\mathbf{2}^{-}-1\right)^{n_{9}} \times \\
& \quad \times\left(\mathbf{3}^{-}-\mathbf{2}^{-}+1\right)^{n_{10}}\left(\mathbf{3}^{-}-\mathbf{1}^{-}+1\right)^{n_{11}}\left(\mathbf{4}^{-}-\mathbf{6}^{-}+\mathbf{8}^{-}\right)^{n_{48}}\left(\mathbf{5}^{-}-\mathbf{7}^{-}+\mathbf{8}^{-}\right)^{n_{58}} \times \\
& \quad \times\left(\mathbf{0}^{+}\right)^{n_{45}} I_{d}\left(n_{1}, n_{2}, n_{3}, n_{4}, n_{5}, 0,0, n_{8}\right), \\
& K_{g}\left(n_{1}, n_{2}, n_{3}, n_{6}, n_{7}, n_{8} ; n_{9}, n_{10}, n_{11} ; n_{68}, n_{78}, n_{67}\right)=\left(\mathbf{1}^{-}+\mathbf{2}^{-}-\mathbf{3}^{-}\right)^{n_{9}} \times \\
& \quad \times\left(\mathbf{3}^{-}-\mathbf{2}^{-}+1\right)^{n_{10}}\left(\mathbf{3}^{-}-\mathbf{1}^{-}+1\right)^{n_{11}}\left(\mathbf{6}^{-}-\mathbf{4}^{-}+\mathbf{8}^{-}\right)^{n_{68}}\left(\mathbf{7}^{-}-\mathbf{5}^{-}+\mathbf{8}^{-}\right)^{n_{78}} \times \\
& \quad \times\left(\mathbf{4}^{-}+\mathbf{5}^{-}-\mathbf{6}^{-}-\mathbf{7}^{-}+\mathbf{0}^{+}\right)^{n_{67}} I_{d}\left(n_{1}, n_{2}, n_{3}, 0,0, n_{6}, n_{7}, n_{8}\right) .
\end{aligned}
$$

\section{Implementation and testing}

The package Grinder consists of a set of mutually recursive procedures for Feynman integrals of various topologies, which reduce a given Feynman integral to simpler ones, until boundary-case integrals with known values are reached. The package is written in REDUCE [23, 24]. Remembering results of previous function calls may make the

program run much faster, if there is enough memory (unfortunately, REDUCE uses linear look-up in remember-tables).

I also re-implemented it in Axiom [25. All expressions involved are linear combinations of basis integrals with coefficients which are rational functions of $d$. It is convenient to use Axiom domain Vector Fraction UnivariatePolynomial, which has all the necessary operations. This makes intermediate expressions shorter than in the case when multivariate rational functions are used for entire expressions, because, typically, not all basis integrals are accompanied by every possible denominator. The amount of GCD calculations is thus reduced. This improvement can be, in principle, back-propagated to the REDUCE implementation by using matrices. However, working with matrices in REDUCE is awkward, because there are no local matrix variables, and no easy way for a function to return a matrix. On the other hand, complete diagram calculations, including tensor and $\gamma$-matrix algebra, can be done in REDUCE.

The main method of testing was checking various recurrence relations (including those which were not directly used for construction of the algorithm) in nested loops over $n_{i}$. For each of two-loop and generalized two-loop integrals, which depend on 5 indices, a typical number of checks was about 20000; each test set of this size runs for a few hours. Integrals with 8 or 9 indices are more difficult to check. Some test sets required a few days of CPU time. A tool showing how many times each linear code segment has been executed would be invaluable for setting up test cases which check all branches at least once. Unfortunately, such a tool is not available in either programming system, and I had to emulate it by hand. 


\section{Acknowledgments}

I am grateful to P.A. Baikov, D.J. Broadhurst, K.G. Chetyrkin, S.A. Larin and J.A.M. Vermaseren for useful discussions, and to INTAS for the grant which allowed me to buy Axiom.

\section{References}

[1] R. Harlander and M. Steinhauser, Automatic computation of Feynman diagrams, Prog. Part. Nucl. Phys. 43 (1999) 167 hep-ph/9812357.

[2] G. 't Hooft and M. Veltman, Regularization and renormalization of gauge fields, Nucl. Phys. B 44 (1972) 189.

[3] F.V. Tkachov, A theorem on analytical calculability of four loop renormalization group functions, Phys. Lett. B 100 (1981) 65;

K.G. Chetyrkin and F.V. Tkachov, Integration by parts: the algorithm to calculate $\beta$ functions in 4 loops, Nucl. Phys. B 192 (1981) 159.

[4] M. Veltman, SCHOONSCHIP, CERN, 1967;

H. Strubbe, Manual for SCHOONSCHIP: a CDC 6000/7000 program for symbolic evaluation of algebraic expressions, Comput. Phys. Commun. 8 (1974) 1.

[5] S.G. Gorishny, S.A. Larin and F.V. Tkachov, Preprint INR P-0330, Moscow, 1984;

S.G. Gorishny, S.A. Larin, L.R. Surguladze and F.V. Tkachov, MINCER: program for multiloop calculations in quantum field theory for the SCHOONSCHIP system, Comput. Phys. Commun. 55 (1989) 381.

[6] S.A. Larin, F.V. Tkachov and J.A.M. Vermaseren, The FORM version of MINCER, preprint NIKHEF-H/91-18, Amsterdam, 1991.

[7] J.A.M. Vermaseren, Symbolic manipulation with FORM, Amsterdam, 1991.

[8] D.J. Broadhurst, Three loop on-shell charge renormalization without integration: $\Lambda_{\mathrm{QED}}^{\overline{\mathrm{MS}}}$ to four loops, Z. Physik C 54 (1992) 599.

[9] J. Fleischer and O.V. Tarasov, SHELL2: package for the calculation of two loop on-shell Feynman diagrams in FORM, Comput. Phys. Commun. 71 (1992) 193.

[10] K.G. Chetyrkin, J.H. Kuhn and M. Steinhauser, Three-loop polarization function and $O\left(\alpha_{s}^{2}\right)$ corrections to the production of heavy quarks, Nucl. Phys. B 482 (1996) 213 hep-ph/9606230.

[11] O.V. Tarasov, Generalized recurrence relations for two-loop propagator integrals with arbitrary masses, Nucl. Phys. B 502 (1997) 455 hep-ph/9703319].

[12] S. Laporta and E. Remiddi, The analytical value of the electron $(g-2)$ at order $\alpha^{3}$ in QED, Phys. Lett. B 379 (1996) 283 hep-ph/9602417. 
[13] K. Melnikov and T. van Ritbergen, The three loop slope of the Dirac form factor and the $1 S$ Lamb shift in hydrogen, Phys. Rev. Lett. 84 (2000) 1673 hep-ph/9911277; The three-loop relation between the $\overline{M S}$ and the pole quark masses, Phys. Lett. $\mathrm{B}$ 482 (2000) 99 [hep-ph/9912391]; The three-loop on-shell renormalization of QCD and $Q E D$, hep-ph/0005131.

[14] M. Neubert, Heavy quark symmetry, Phys. Rep. 245 (1994) 259 hep-ph/9306320;

A.V. Manohar and M.B. Wise, Heavy quark physics, Cambridge University Press, Cambridge 2000.

[15] A.G. Grozin, Introduction to the heavy quark effective theory, 1, hep-ph/9908366; Recent progress on the HQET lagrangian, in Proc. IV int. workshop on progress in heavy quark physics, M. Beyer, T. Mannel and H. Schröder eds., Univ. of Rostock, 1998 [hep-ph/9711269].

[16] D.J. Broadhurst and A.G. Grozin, Two-loop renormalization of the effective field theory of a static quark, Phys. Lett. B 267 (1991) 105 hep-ph/9908362.

[17] D.J. Broadhurst and A.G. Grozin, Multiloop calculations in heavy quark effective theory, in New computing technique in physics research IV, B. Denby and D. Perret-Gallix eds., World Scientific, Singapore 1995, p. 217 hep-ph/9504400.

[18] S.A. Larin, private communication.

[19] M. Beneke and V.M. Braun, Heavy quark effective theory beyond perturbation theory: renormalons, the pole mass and the residual mass term, Nucl. Phys. B 426 (1994) 301 hep-ph/9402364.

[20] K.G. Chetyrkin, A.L. Kataev and F.V. Tkachov, New approach to evaluation of multiloop Feynman integrals: the Gegenbauer polynomial $x$ space technique, Nucl. Phys. B 174 (1980) 345.

[21] D.J. Broadhurst, On the enumeration of irreducible $k$-fold Euler sums and their roles in knot theory and field theory, hep-th/9604128; Conjectured enumeration of irreducible multiple zeta values, from knots and Feynman diagrams, hep-th/9612012;

J.M. Borwein, D.M. Bradley and D.J. Broadhurst, Evaluations of $k$-fold Euler/Zagier sums: a compendium of results for arbitrary $k$, Electronic J. Combinatorics 4(2) (1997) R5 hep-th/9611004.

[22] P.A. Baikov, The criterion of irreducibility of multiloop Feynman integrals, Phys. Lett. B 474 (2000) 385 hep-ph/9912421.

[23] A.C. Hearn, REDUCE user's manual, Version 3.7, 1999.

[24] A.G. Grozin, Using REDUCE in high energy physics, Cambridge University Press, Cambridge, 1997.

[25] R.D. Jenks and R.S. Sutor, Axiom: the scientific computation system, Springer-Verlag, 1992. 